This document is the accepted manuscript version of the following article: Merg, A. D., Touponse, G., van Genderen, E., Blum, T. B., Zuo, X., Bazrafshan, A., ... Conticello, V. P. (2020). Shape-shifting peptide nanomaterials: surface asymmetry enables pHdependent formation and interconversion of collagen tubes and sheets. Journal of the American Chemical Society, 142(47), 19956-19968. https://doi.org/10.1021/jacs.0c08174

\title{
Shape-Shifting Peptide Nanomaterials: Surface Asymmetry Enables pH-Dependent Formation and Interconversion of Collagen Tubes and Sheets
}

\author{
Andrea D. Merg,,+ Gavin Touponse,,$+\dagger$ Eric van Genderen, ${ }^{\ddagger}$ Thorsten B. Blum, ${ }^{\ddagger}$ Xiaobing Zuo,, Alisina \\ Bazrafshan, ${ }^{\dagger}$ Hew Ming Helen Siaw, ${ }^{\dagger}$ Arthur McCanna, ${ }^{\perp}$ R. Brian Dyer, ${ }^{\dagger}$ Khalid Salaita, ${ }^{\dagger}$ Jan Pieter \\ Abrahams, ${ }^{\ddagger}$, and Vincent P. Conticello*,†
}

†Department of Chemistry, Emory University, Atlanta, Georgia 30322, United States

₹Paul Scherrer Institut, CH-5232 Villigen, Switzerland

§X-ray Science Division, Argonne National Laboratory, Lemont, Illinois 60439, United States

${ }^{\perp}$ The Robert P. Apkarian Integrated Electron Microscopy Core, Emory University, Atlanta, GA, 30322, United States

ॠCenter for Cellular Imaging and NanoAnalytics, Biozentrum, University of Basel, CH-4058 Basel, Switzerland

peptide self-assembly, collagen, $\mathrm{pH}$ responsive, shape-shifting, biomolecular assembly, tubes, nanosheets

\begin{abstract}
The fabrication of dynamic, transformable biomaterials that respond to environmental cues represents a significant step forward in the development of synthetic materials that rival their highly functional, natural counterparts. Here, we describe the design and synthesis of crystalline supramolecular architectures from charge-complementary heteromeric pairs of collagen-mimetic peptides (CMPs). Under appropriate conditions, CMP pairs spontaneously assemble into either 1D ultraporous (pore diameter $>100 \mathrm{~nm}$ ) tubes or 2D bilayer nanosheets due to the structural asymmetry that arises from heteromeric self-association. Crystalline collagen tubes represent a heretofore unobserved morphology of this common biomaterial. In-depth structural characterization from a suite of biophysical methods, including TEM, AFM, high-resolution cryo-EM, and SAXS/WAXS measurements, reveals that the sheet and tube assemblies possess a similar underlying lattice structure. The experimental evidence suggests that the tubular structures are a consequence of the self-scrolling of incipient 2D layers of collagen triple helices, and that the scrolling direction determines the formation of two distinct structural isoforms. Furthermore, we show that nanosheets and tubes can spontaneously interconvert through manipulation of the assembly $\mathrm{pH}$ and systematic adjustment of the CMP sequence. Altogether, we establish initial guidelines for the construction of dynamically responsive 1D and 2D assemblies that undergo a structurally programmed morphological transition.
\end{abstract}

\section{INTRODUCTION}

The controlled fabrication of functionally responsive assemblies remains a key contemporary challenge in nanoscience. While sequence-defined polymers (e.g., peptides, nucleic acids, and proteins) are promising building blocks for constructing a myriad of assemblies with excellent nanoscale order, ${ }^{1-4}$ the ability to reliably and predictably encode responsive behavior and higher-order function lies beyond current synthetic capabilities, especially when compared to multicomponent macromolecular machines of living systems. ${ }^{5-6}$

Most synthetic peptide assemblies are derived from homomeric self-association of appropriately designed, singlecomponent protomers. However, the structural information required to drive selective self-assembly must necessarily be encoded within a single peptide sequence. While this approach has been incredibly successful, structural evolution can be difficult to control due to over- design of the corresponding peptide sequences. Heteromeric self-association affords an alternative approach to define higher order structure among peptide-based materials. Peptide sequence space can be expanded through use of two or more appropriately designed and selectively interacting protomers. This approach presents the opportunity to encode a greater amount of structural information within the collective ensemble of peptide sequences, and enables the design and construction of supramolecular assemblies having greater structural and functional complexity. ${ }^{7-23}$ In addition, the individual peptide sequences within the protomer pool can be chemically modified to further increase the structure complexity of the resultant assemblies.

While heteromeric self-association has been employed successfully in the design of synthetic peptide assemblies, the engineering of responsive behavior remains a significant hurdle to implementation of these peptide-based materials in tailored applications. ${ }^{24}$ The promise of this ap- 
proach can be glimpsed through comparison with DNA nanotechnology, in which multiple sequence-specific promoters are employed that self-assemble with high structural specificity into structurally defined supramolecular assemblies.1, 25 Competition between different oligonucleotides for a complementary partner enables engineering of precise responsive behavior at the molecular level. Oligonucleotides have the advantage that the Watson-Crick base pairing rules provide a digital scoring function that can encode higher order structure and dynamic function in a predictive manner. ${ }^{26}$ In contrast, the rules that govern interactions between peptides and proteins-and their effect on higher order structure-are more complex and difficult to reliably predict.

Here, we describe the design of a heteromeric pair of charge-complementary collagen-mimetic peptides (CMPs) that self-assemble into either ultraporous laminated tubes or bilayer nanosheets. CMPs represent a highly versatile and programmable biomimetic assembly building unit. ${ }^{27-38}$ We demonstrate that systematic changes to the sequence design and assembly conditions can toggle the peptide assemblies between the two structurally distinct polymorphs. This morphological transition is triggered through a change in $\mathrm{pH}$ and rationalized in terms of the influence of surface charge on the stacking of crystalline layers of collagen-mimetic triple helices. The responsive behavior of these heteromeric assemblies provides a rubric for the molecular design of shape-shifting nanomaterials that can enhance the potential utility of these materials for applications that include drug delivery, controlled release, gated catalysis, etc. ${ }^{39}$

\section{RESULTS AND DISCUSSION}

Recently, we developed protocols for the design and construction of nanosheets from crystallizable collagen triple helices. ${ }^{40-44}$ The triple helices, which serve as rigid, rod-like tectons, comprise CMP sequences having positively charged, neutral, and negatively charged segments (Figure 1a). This triblock architecture promotes ordered selfassembly into a 2D lattice via electrostatic interactions between antiparallel-oriented triple helical protomers (Figure 1b). The packing arrangement is consistent with crystal structures of CMPs deposited in the Protein Data Bank (PDB), in which the majority have been shown to crystallize in layered structures in an antiparallel orientation. ${ }^{45-47}$ We postulated that the triblock architecture of these CMPs adventitiously reinforces this native packing arrangement.

Based on the previous sequence design, in which triple helical units possess both positively and negatively charged segments, we surmised that these charged segments could be deconstructed into two separate peptide molecules (Figure 1c). In this manner, heteromeric pairs of CMP triple helices, one having positively charged triads and the other having negatively charged triads could be coassembled to form hierarchical peptide-based architectures. Both peptides possess six repeats of the canonical collagen triad, Pro-Hyp-Gly; the presence of which has been demonstrated to stabilize the triple helical structure. In order to maintain the favorable antiparallel packing arrangement between triple helices, the positively and negatively charged segments are placed at the $\mathrm{N}$ - and Ctermini, respectively, of the heteromeric pairs of peptides (Figure 1c). We hypothesized that these complementary charged CMP pairs would ultimately assemble into 2D "Janus-like" lamellar structures displaying surface asymmetry (Figure 1c). For 2D assemblies, the two faces of the sheet would be physically and chemically distinguishable, which was not achievable using our previous CMP molecular designs.

\section{a}

$$
\begin{aligned}
& \mathbf{C M P}=(\text { Pro-Y-Gly })_{a}-(\text { Pro-Hyp-Gly })_{b}-(\mathbf{X}-\text { Hyp-Gly })_{c}= \\
& Y=\text { positively charged amino acid }
\end{aligned}
$$

b

$\mathrm{X}=$ negatively charged amino acid
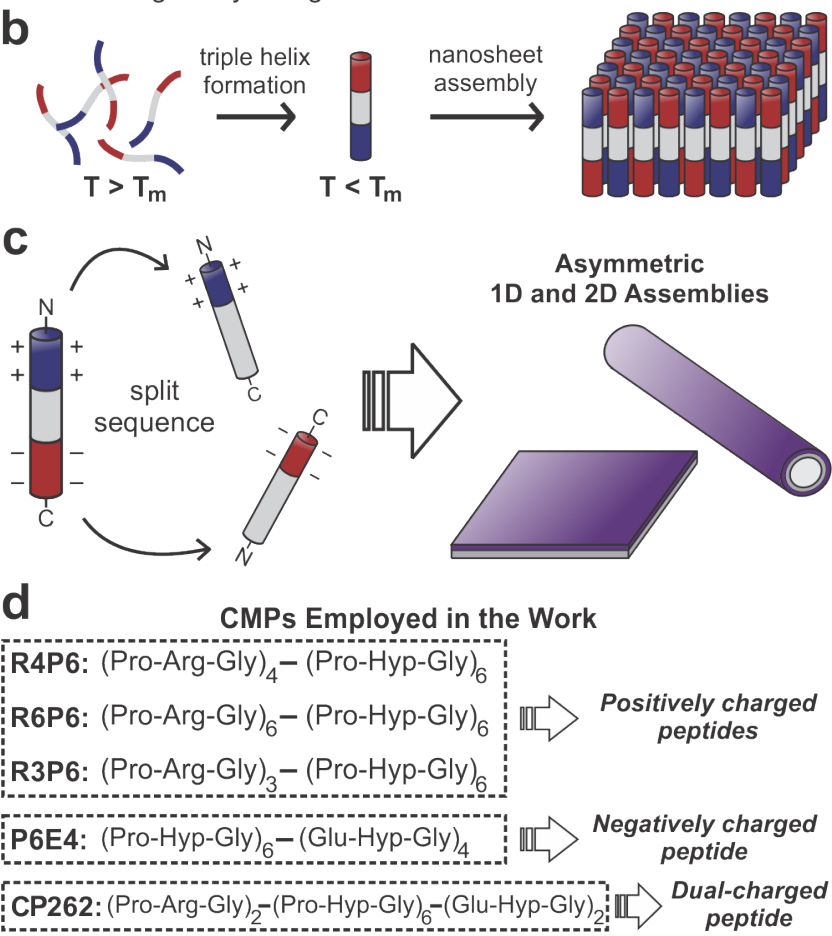

Figure 1. (a) Amino acid sequence of a self-complementary CMP having a triblock architecture with sequential positively charged, neutral, and negatively charged triad segments. (b) Folding of synthetic CMPs into self-complementary collagen triple helices upon cooling the assembly solution below the $T_{\mathrm{m}}$ triggers spontaneous assembly into a 2D lattice. CMP triple helices pack in an antiparallel fashion to facilitate favorable charge interactions between structurally adjacent triple helices. (c) Deconstruction of the triblock sequence architecture into two oppositely charged homomeric collagen triple helices as a means to construct asymmetric 1D and/or 2D assemblies. (d) List of CMP sequences employed within this study.

Two peptide sequences, termed R4P6 and P6E4 (Figure 1d), were synthesized using standard solid-phase peptide synthesis protocols and characterized via MALDI-TOF mass spectrometry (Figure S1). Both CMPs consist of a diblock architecture having six Pro-Hyp-Gly triads attached to a charged block consisting of four positively or negatively charged triads containing arginine (R4P6) or glutamic acid (P6E4) residues, respectively. The sequences were designed such that co-assembly of the pre-formed homomeric triple helices would permit the antiparallel packing arrangement to be maintained (vide supra). Fur- 
thermore, we anticipated that co-assemblies derived from both peptides would most likely occur under neutral $\mathrm{pH}$ conditions, which would give rise to favorable Coulombic interactions. Tables S1 and S2 show estimated pI values of individual peptides as well as approximate $\mathrm{pI}$ values for equimolar mixtures, respectively. We note that these estimated $\mathrm{pI}$ values serve only as a reference point to contextualize subsequent structural studies. The true charge state of the respective peptides reflects not only the solution conditions employed for peptide assembly, but also the triple-helical structure of CMPs. Both considerations may result in deviation of the estimated pI values from the actual pI values.

Before conducting the heteromeric assembly experiments, we characterized both CMPs independently. Circular dichroism (CD) spectropolarimetry of solutions of the single-component R4P6 and P6E4, collected after one week of incubation at $4{ }^{\circ} \mathrm{C}$, confirm that the respective CMPs form collagen triple helices, as determined by the presence of a positive maximum at ca. $224 \mathrm{~nm}$ and a negative minimum at ca. $198 \mathrm{~nm}$ (Figure 2a). To assess the stability of the homomeric triple helices, Rpn values, which report the ellipticity ratio of the positive and negative maximum intensities, were calculated. ${ }^{48} \mathrm{Rpn}$ values of 0.081 and 0.064 for R4P6 and P6E4, respectively, indicate that the R4P6 displays a greater propensity to adopt a triple helical conformation than its negatively charged counterpart. Furthermore, CD thermal denaturation studies confirm this difference in triple helix stability between the two homomeric triple helices $\left(T_{\mathrm{m}}(\mathbf{R 4 P 6})=37{ }^{\circ} \mathrm{C}, T_{\mathrm{m}}(\mathbf{P 6 E 4})=\right.$ $28{ }^{\circ} \mathrm{C}$; Figure $\mathbf{2 b}$ ). These results agree with prior reports that presented Pro-Arg-Gly triads as a highly stable complement to Pro-Hyp-Gly, in comparison to Glu-Hyp-Gly. ${ }^{49-50}$
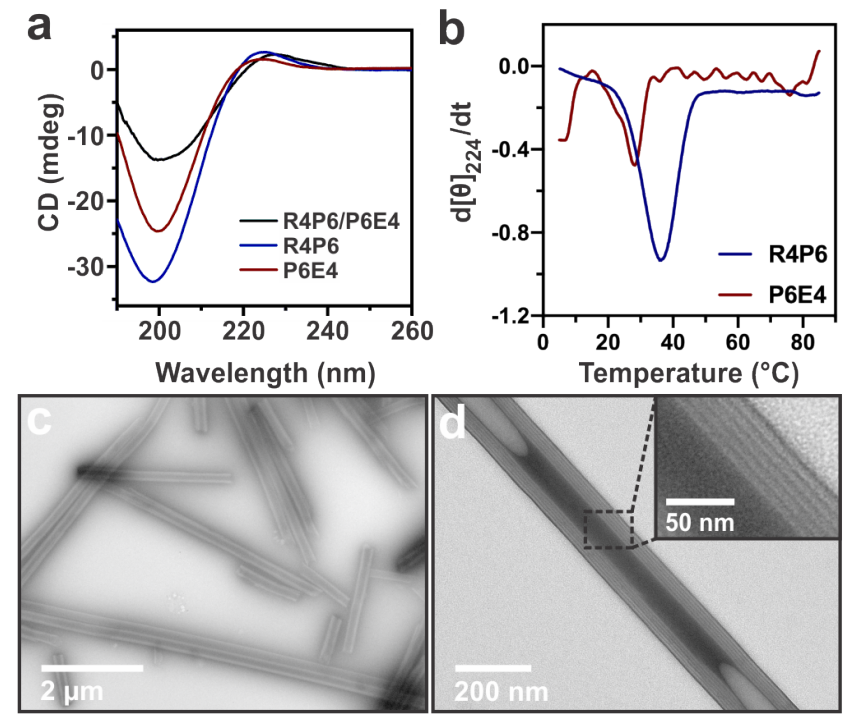

Figure 2. R6P6/P6E4 assembly characterization. (a) CD spectra of R4P6, P6E4, and R4P6/P6E4 tubes ([peptide] = 1 $\mathrm{mg} / \mathrm{mL}$ ) in MOPS buffer (20 mM, pH 7.0) after one week of incubation at ambient temperature. Rpn values of 0.081 , 0.064, and 0.12 were obtained for R4P6, P6E4, and R4P6/P6E4, respectively. (b) First derivative of the CD signal at $224 \mathrm{~nm}$ as a function of temperature for R4P6 and P6E4 (1 $\mathrm{mg} / \mathrm{mL}$ ). (c) Stained TEM image of R4P6 tubes ([total peptide $]=1 \mathrm{mg} / \mathrm{mL})$ assembled in MOPS buffer $(20 \mathrm{mM}, \mathrm{pH} 7.0)$ after one week of assembly time. (d) TEM image revealing lamination of concentric layers comprising the tube walls.

Based on previous investigations in our laboratory, we concluded that the slow, controlled formation of CMP triple helices would be necessary for fabrication of welldefined nanosheets. ${ }^{40}$ Concerned about the formation of kinetically trapped aggregates arising from mixing preformed R4P6 and P6E4 triple helices, we decided to employ a protocol that closely mimicked the previously reported assembly conditions. Stock solutions of R4P6 (1 $\mathrm{mg} / \mathrm{mL}$ ) triple helices were prepared by thermally annealing R4P6 in MOPS buffer (20 mM, pH. 7.0) followed by incubation for at least one week at $4{ }^{\circ} \mathrm{C}$ to maximize triple helix formation (see Experimental Methods; Figure S2). P6E4 solutions in identical buffer conditions were thermally annealed and cooled to ambient temperature. This freshly annealed P6E4 solution was mixed with an equivalent volume of the R4P6 stock solution. Minutes after mixing, the solution became cloudy, which suggested the presence of supramolecular assembles.

Surprisingly, co-assembly of R4P6 and P6E4 triple helices using this procedure yields tube structures rather than nanosheets. The corresponding tubes span tens of microns in length and hundreds of nanometers in diameter, as evidenced from transmission electron microscopy measurements (TEM; Figure 2c). Negative staining clearly indicates differential contrast between the inner lumen and outer walls of the tubes (Figure 2d). Furthermore, striations within the tube walls suggest that the tubes consist of stacked concentric lamellae (Figure 2d inset). No assembled structures are observed from annealed solutions of the individual homomeric triple helices, which confirms that assemblies only occur when both CMP triple helix pairs are present (Figure S3). Furthermore, one-pot annealing of R4P6 and P6E4 yield unordered aggregates, indicating that the formation of homomeric triple helices is an essential pre-requisite for hierarchical assembly (Figure S4). The Rpn value of 0.12 for the R4P6/P6E4 tubes indicates that the triple helices are substantially more stable when co-assembled with their complementary charged partners (Figure 2a). The added stability is attributed to close packing of oppositely charged triple helices in an antiparallel orientation, which would counteract the electrostatic repulsion between adjacent strands within isolated CMP triple helices of the individual component peptides. Unfortunately, CD thermal denaturation studies could not be effectively applied to solutions of the tubes due to limited solubility in near-neutral $\mathrm{pH}$ buffers.

These results contrast with previously published reports from our laboratory, in which CMPs having a selfcomplementary triblock architecture were observed to preferentially self-assemble into nanosheets. We ascribe this difference in morphology to the antiparallel packing of R4P6 and P6E4 triple helices, which, upon co-assembly, would result in an incipient 2D lattice with surface asymmetry. The larger effective packing diameter of the charged residues that comprise one side of the nanosheet could induce curvature to the extended 2D structure, and, thereby, lead to the formation of tubular assemblies. To test this hypothesis, a symmetric variant, CP262 (Figure 1d), was synthesized and purified (Figure S5). CP262 possesses an 
identical number of triads to R4P6 and P6E4 but differs in that it contains both positive- and negative-charged segments. TEM images confirm that CP262 assembles into 2D structures (Figure S6), thereby suggesting that the asymmetry associated with antiparallel-packed R4P6 and P6E4 triple helices is responsible for tube formation.

Although the R4P6/P6E4 tubes appear to adopt a welldefined structure, they were prone to aggregation through lateral association at near-neutral $\mathrm{pH}$. To improve colloidal stability, a longer, positively charged CMP was designed and synthesized (R6P6; Figure 1d). We hypothesized that the two extra positively charged triads would extend from the tube surface and prevent self-association between tubes in solution due to electrostatic repulsion at nearneutral $\mathrm{pH}$. This design concept was used successfully for constructing monolayer nanosheets from triblock CMPs. ${ }^{41}$ Like R4P6, R6P6 forms stable collagen triple helices after approximately 1 week in buffered aqueous solutions $(\mathrm{pH}$ 6-8; Figure S7). Rpn values for buffered aqueous solutions of R6P6 in the near-neutral pH range are consistent with the value calculated for R4P6 triple helices (Rpn ca. 0.08; Figure S7d). TEM images of R6P6 solutions confirm that no discernable assemblies form in the absence of the complementary triple helical partner (Figure S8).

R6P6/P6E4 co-assembly experiments were conducted using the same protocol described above. TEM imaging of the resultant assemblies reveal that the morphology unexpectedly depended on the $\mathrm{pH}$ of the assembly buffer. Under acidic and neutral conditions in MES buffer $(20 \mathrm{mM}$, $\mathrm{pH}$ 6.0) and MOPS buffer (20 mM, pH 7.0), respectively, sheet-like assemblies are observed (Figures 3a and S9). In contrast, large-diameter, multi-walled tubes are formed exclusively at basic pH in TAPS buffer (20 mM, pH 8.0) (vide infra).

The R6P6/P6E4 nanosheets are similar to previously studied nanosheets derived from self-complementary triblock CMPs. However, unlike prior reports, where sheets typically exhibited square or rectangular dimensions, the R6P6/P6E4 sheets frequently display a parallelogram-like morphology (Figure 3a inset). Atomic force microscopy (AFM) height profiles reveal a sheet thickness of ca. $17 \mathrm{~nm}$. This heightvalue suggests that the sheets possess a bilayer structure, as it is approximately double the expected thickness of $10 \mathrm{~nm}$ for a monolayer sheet (Figure $\mathbf{3 b}$ ). The smaller height value compared to the theoretical value of bilayer sheets (ca. $20 \mathrm{~nm}$ or twice the length of R6P6 triple helix) is attributed to the dry imaging conditions, which would collapse the extra charged triads that extend from the sheet surface. ${ }^{41}$ The proposed bilayer structure is further supported by TEM images, which reveal differences in electron contrast at the periphery of the assembly suggesting the presence of two distinct layers (Figure S10).

Synchrotron small- and wide-angle X-ray scattering (SAXS/WAXS) measurements of R6P6/P6E4 nanosheets provide additional evidence of the proposed bilayer architecture. A Guinier analysis for sheet-like forms, returned a sheet thickness value of $230 \pm 5 \AA$ (Figure S11). Similarly, oscillations in the form factor scattering could be fit directly with a lamellar model, which afforded a sheet thickness value of $218 \pm 26 \AA$ (Figure S12). Both measurements are internally consistent and in agreement with the expected thickness of a bilayer assembly architecture. We ascribe the discrepancy in sheet thickness between SAXS and AFM measurements to the different experimental conditions under which data were collected. Solution SAXS measurements detect the presence of the hydrated extensions corresponding to the two extra charged triads of R6P6 triple helices, which would be collapsed under the dry imaging conditions employed in AFM height measurements. We have observed similar distinctions between SAXS thickness and AFM height measurements for related collagen nanosheet samples. ${ }^{41}$
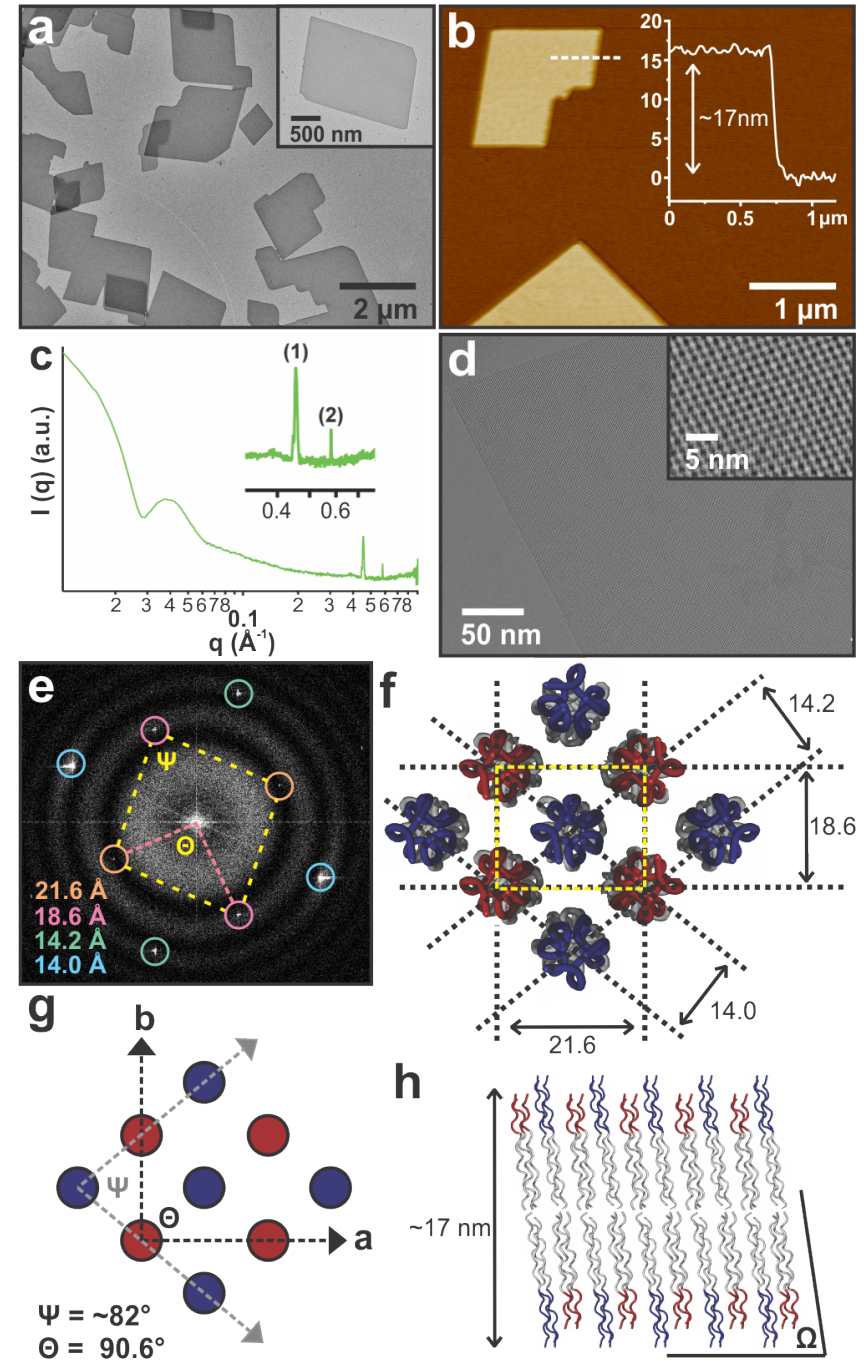

Figure 3. R6P6/P6E4 nanosheet characterization. (a) Stained TEM images of R6P6/P6E4 nanosheets ( $1 \mathrm{mg} / \mathrm{mL})$ assembled in MOPS buffer (20 mM, pH 7.0). (b) AFM image and height trace of R6P6/P6E4 nanosheets. (c) Synchrotron SAXS/WAXS scattering profile of R6P6/P6E4 nanosheets (4 $\mathrm{mg} / \mathrm{mL}$ ) in MOPS buffer (20 mM, pH 7.0). Peaks (1) and (2) correspond to $d$-spacings of $13.8 \AA$ and $10.8 \AA$, respectively. (d) Overlay of the lattice-filtered cryo-EM image over its respective driftcorrected image (Figure S15a) of a R6P6/P6E4 nanosheet. Inset: high magnification image of the ordered 2D lattice comprising the nanosheets. (e) Representative FFT analysis of R6P6/P6E4 nanosheets. Averaged $d$-spacings values, obtained from analysis of several nanosheets, are listed for the 2D lattice. (f) Packing model of collagen triple helices within the 2D lattice using averaged $d$-spacing values (in Angstroms) from FFT analysis. Red and blue coloring denotes negative 
and positive triads at the surface to highlight the antiparallel packing between adjacent triple helices. The yellow box highlights the monoclinic unit cell. (g) 2D lattice packing model denoting the angles $\Theta$ and $\psi$, which are ca. $82^{\circ}$ and $90.6^{\circ}$, respectively. (h) Side view of the proposed model of R6P6/P6E4 nanosheets revealing the bilayer structure of collagen layers, and their tilted orientation with respect to the surface. The tilted layers are packed "back-to-back", in which charged segments interface with the aqueous media.

Two Bragg diffraction peaks, labeled (1) and (2) (Figure 3c), are observed in the high $q$ region of the SAXS/WAXS intensity profile and correspond to $d$-spacings of ca. $13.8 \AA$ and ca. $10.8 \AA$, respectively. These values are similar to $d$ spacing values that we have reported for crystalline $2 \mathrm{D}$ lattices of collagen nanosheets (Figure S13). ${ }^{40-44}$ The relationship between the two measured values indicate that the lattice deviates from a tetragonal lattice $(10.8 \times \sqrt{2} \neq$ 13.8) and represents the most structurally distorted 2D lattice that we have thus far observed for synthetic collagen nanosheets.

Cryo-EM measurements were performed to further characterize the internal structure of the $2 \mathrm{D}$ assemblies. Filtered cryo-EM micrographs provide striking visual evidence of the ordered 2D lattice that constitutes the nanosheet assemblies (Figure 3d). Representative Fast Fourier transform (FFT) reveals a two-fold rotational symmetry that confirms the parallelogram crystal habit that we observed from negative-stain TEM measurements (Figures 3e and S14). Bragg spots, with resolution of up to $2.7 \AA$ signify the high degree of crystallinity (Figure S14). Figure 3e shows average $d$-spacing values from FFT analysis of several crystals. The 2D crystal is best described as having a monoclinic unit cell with lattice parameters $\mathrm{a}=$ $21.6 \AA$ and $b=18.6 \AA$, which are associated with the $[1,0]$ and $[0,1]$ lattice planes, respectively (Figures 3e-g and S15 and Table 1). In addition, two pairs of Bragg spots, which correspond to $d$-spacings of $14.2 \AA$ and $14.0 \AA$, define the distances between lattice planes having Miller indices of $[1,1]$ and $[1,-1]$, respectively (Figures 3e-g and S15 and Table 1). The lattice parameter values differ slightly from those observed from SAXS analysis. In the latter case, only a subset of the diffraction peaks observed in the cryo-EM analysis could be detected in the SAXS intensity profile. The lattice spacing corresponding to the distances of the $[1,1]$ and $[1,-1]$ lattice planes were not resolved in the SAXS data and were observed at a slightly shorter distance (13.8 $\AA$ ). In comparison, the $d$-spacing for the $[2,0](10.8 \AA=21.6 \AA / 2)$ is the same for both SAXS and cryo-EM. The discrepancy in the observed metrical values between measurements may arise due to differences in conditions under which the measurements were acquired.

Finally, FFT data provides real-space angular information that relates to the unit cell and crystal habit ( $\psi$ and $\Theta$; Figure $3 \mathbf{e}, \mathbf{g})$. The angle between the $\mathrm{a}$ and $\mathrm{b}$ vectors $([1,0]$ and $[0,1]$ planes $), \Theta$, is approximately $90^{\circ}\left(90.6^{\circ}\right.$; Figure 3e,g). In contrast, the internal angle between the $[1,1]$ and $[1,-1]$ planes, $\psi$, is approximately $82^{\circ}$, which is consistent with the internal angle measured from the intersection of edges in parallelogram-shaped crystals (Figure S16). This confirms that the parallelogram crystal habit is derived from the monoclinic unit cell (Figures 3f,g).
Based on analysis of the structural information gathered from AFM, SAXS/WAXS, and cryo-EM measurements, we propose the following model for the R4P6/P6E4 nanosheet assemblies. Within a single 2D layer, oppositely charged triple helical protomers are packed in an antiparallel orientation within a distorted tetragonal lattice (Figure 3f). The nanosheets comprise two such CMP layers that are packed "back-to-back", which allows the charged segments to interact with the aqueous medium at the exposed surfaces of the bilayer assemblies (Figure $\mathbf{3 h}$ ). The proposed bilayer structure schematically resembles the double leaflet architecture of lipid bilayer membranes, in which the charged head groups are exposed at the surface and the hydrophobic tails congregate within the core. Furthermore, we propose that the triple helices are tilted at an angle with respect to the assembly surface ( $\Omega$; Figure $\mathbf{3 h}$ ) on the basis of information from unprocessed cryo-EM images that appear to show parallel "lines" across the nanosheet surface (Figure S17). Further evidence of this tilting is observed from cryo-EM analysis of the tube samples, which provides a "side-view" of the triple helices with respect to the electron beam (vide infra).

In contrast, the R6P6/P6E4 assemblies observed at basic $\mathrm{pH}$ values are similar in morphology to the tubular assemblies of R4P6/P6E4 under neutral pH conditions (Figure 4a,b). The tube walls consist of multiple layers with an approximate thickness of $10 \mathrm{~nm}$ (Figure S18). This distance correlates well with the theoretical length of the longer CMP triple helices of R6P6 (36 residues x 0.286 $\mathrm{nm}=10.3 \mathrm{~nm}),{ }^{51-52}$ thereby suggesting that the tube walls are composed of laminated layers of CMP triple helices. Scanning electron microscopy (SEM) verifies the hollow interior of the assemblies and reveals that the tube surface consists of areas with varying number of layers along the contour length of the assemblies (Figure 4c and S19). In addition, AFM height and amplitude traces indicate that the structures retain their cylindrical shape under dry imaging conditions, which suggests that the tubes are highly rigid, presumably due to the reinforcement of the tubular structure through lamination of multiple layers of collagen triple helices (Figure 4d,e). Line scans along the tube axis reveal that the height of the surface layers are approximately $10 \mathrm{~nm}$, which is consistent with the thickness measurements of the individual layers of the tubes (Figure $\mathbf{4 f}, \mathbf{g}$ ). These results demonstrate that the number of layers could differ along the length of the tube, including between diametrically opposite tube walls. We speculate that these tubes arise from scrolling of incipient asymmetric (i.e., Janus) nanosheets, although microscopy measurements have not been able to directly capture this process (vide infra).

Synchrotron SAXS/WAXS measurements were employed to analyze the structure of collagen tubes in solution (Figure 4h). Distinct features are observed in the intensity profile on three different length scales. At low $q$ values (ca. $0.005-0.02 \AA^{-1}$ ), the form factor scattering could be simulated in terms of a hollow cylinder with polydispersity in the inner radius $(95 \pm 12 \mathrm{~nm})$ and wall thickness $(37 \pm 4$ $\mathrm{nm}$ ) (Figure S20). These values roughly agree with the TEM data in that tubes with varying degrees of lamination were observed that would result in cross-sectional 

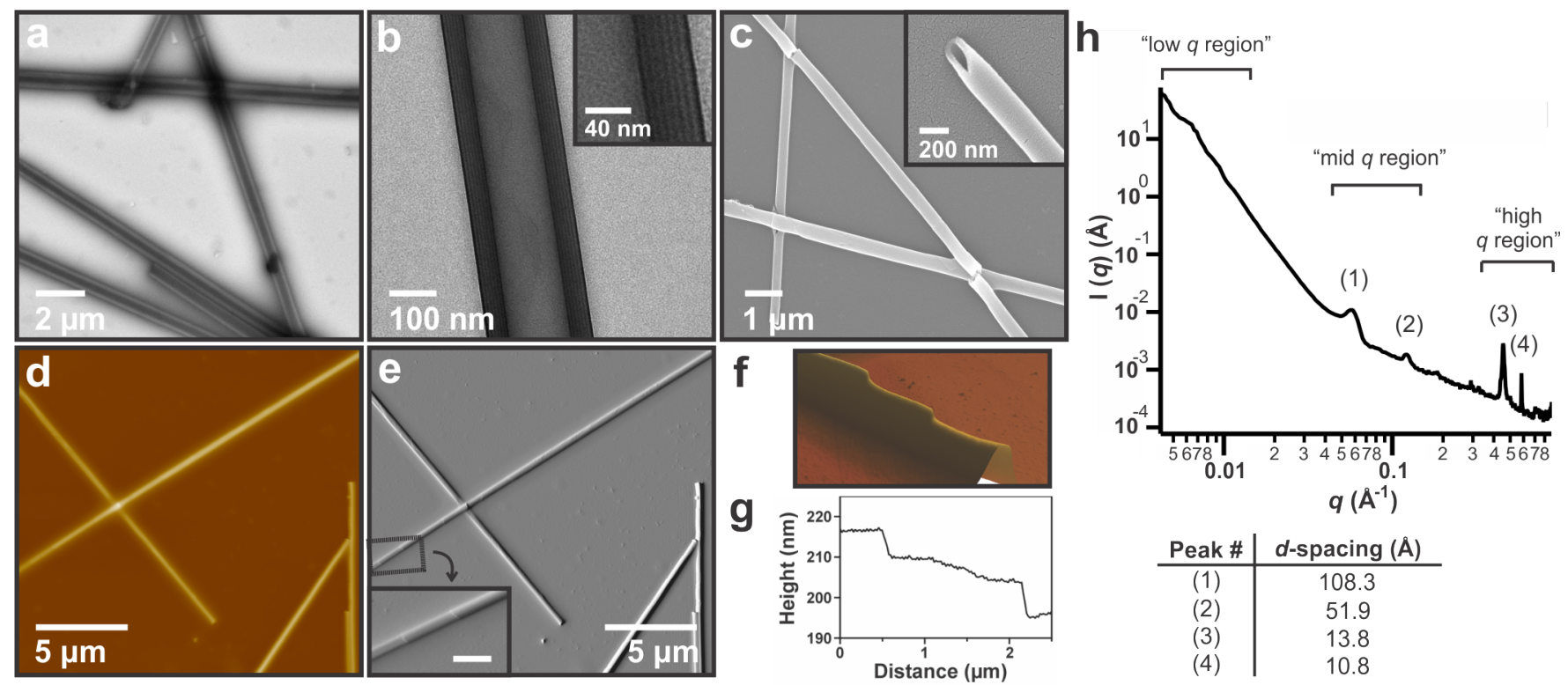

Figure 4. Microscopic analysis of R6P6/P6E4 tubes. (a) Negatively stained TEM image of R6P6/P6E4 tubes (1 mg/mL) assembled in TAPS buffer (20 mM, pH 8.0). (b) High-magnification TEM image revealing the lamellar structure that comprises the tube walls. (c) SEM images of R6P6/P6E4 tubes. AFM (d) height trace and (e) amplitude trace of R6P6/P6E4 tubes deposited onto mica. Inset scale bar $=1 \mu \mathrm{m}$. (f) 3D reconstruction from AFM data revealing the change in height along the tube shown in the inset of (e). (g) AFM height trace along the tube shown in (f). (h) Synchrotron SAXS/WAXS scattering profile of R6P6/P6E4 tubes (2 $\mathrm{mg} / \mathrm{mL}$ ) in TAPS buffer (20 mM, pH 8.0).

polydispersity. In the mid $q$ region (ca. $0.04-0.15 \AA^{-1}$ ), two relatively broad peaks, labeled (1) and (2), are observed at approximate $q$ values of $0.0579 \AA^{-1}$ and $0.122 \AA^{-1}$, respectively. We posit that these peaks correspond to the lamellar stacking periodicity for the concentric scrolled layers, as the stronger peak (peak (1)) corresponds to a $d$-spacing of ca. $108 \AA$, which coincides within experimental error to the thickness of layers observed from TEM images of the tubes and the theoretical length of a collagen triple helix (vide supra). Peak (2) likely corresponds to a second harmonic of the first peak ( $d$-spacing of ca. $52 \AA$ ). The absence of a peak corresponding to 2 x peptide length (ca. $216 \AA$ ), as observed for the bilayer sheets, suggests that the layers within the tubes are packed in an asymmetric "face-toback" orientation rather than an alternating "face-toface/back-to-back" orientation of the bilayer nanosheets.

Finally, peaks (3) and (4), found at high $q$ values, are associated with the crystalline 2D lattice within layers of packed triple helical CMPs (Figure 4h). These peaks, which correspond to $d$-spacings of $13.8 \AA$ and $10.8 \AA$, respectively, are identical to the lattice packing parameters obtained from SAXS data of the sheet sample, which suggests that the sheets and tubes possess the same underlying 2D layered structure of collagen triple helices. These results imply that the formation of tube versus sheet assemblies for the R6P6/P6E4 system is not due to differences in the internal 2D arrangement of triple helices, but rather to the effect of assembly conditions. Although a detailed analysis was not performed, the general features of the synchrotron SAXS/WAXS intensity profile of the R4P6/P6E4 tubes was observed to be similar to that of the R6P6/P6E4 tubes over all three length-scales, albeit with slight differences in metrical parameters (Figure S21).

Cryo-EM was employed to interrogate the internal structure of the tubular assemblies at high-resolution (Figure 5a-c). Unexpectedly, analysis of cryo-EM images indicated the presence of two structurally distinct populations of tubes: thin tubes (R6T_thin), having 2 or 3 layers ( $\mathrm{n}=2,3$ ), and thick tubes (R6T_thick), having 4 or more layers (n $\geq$ 4) (Figure 5b,c). The distinction between the two different tube populations is manifested in terms of a sharp discontinuity in inner diameter distance that is observed between tubes having three layers and four layers (Figure 5d).

Cryo-EM images of the representative tubes from the two classes indicate that the orientation of triple helices within the layers differs between the two tube populations. The angle of the triple helix with respect to the central tube axis (denoted $\Phi$ in Figure $\mathbf{5 b}, \mathbf{c}$ ) is different for R6T_thick $\left(\Phi \approx 90^{\circ}\right)$ and R6T_thin $\left(\Phi \approx 82^{\circ}\right)$ tubes. Furthermore, cryo-EM image analysis reveals discrepancies between repeat distances $(s)$ along the tube axis $(s=18.5 \AA$ and $21.5 \AA$ for R6T_thin and R6T_thick tubes, respectively). Layer thickness values, $r$, are similar $(r=11.1$ and 10.4 $\mathrm{nm}$ for both tubes, respectively) and correlate with the TEM and SAX/WAXS data. FFT analysis of the tubes reveals spots associated with diffraction related to the Moiré pattern that arises from interference between the two sides of the respective tubes. ${ }^{53}$ Lattice spacings can be calculated from the FFT analysis, which provide distances of $21.5 \AA$ and $18.5 \AA$ for the unit cell parameters of R6T_thin and $21.5 \AA$ and $19.5 \AA$ for the unit cell parameters of R6T_thick tubes (Table 1). These distances reveal slight differences in the packing arrangement of triple helices within the layers that comprise the two respective populations of tubes. The $\Theta$ values, calculated for the respective unit cells of each class of tubes, remain at ca. $90.6^{\circ}$. The calculated values of the metrical parameters for R6P6/P6E4 sheets, R6T_thin tubes, and R6T_thick tubes are compiled in Table 1 . Based on this analysis, we conclude that the underly- 
ing 2D triple helix lattice for the two populations of tubes is similar to the 2D lattice that constitutes the nanosheets (Figure 5e).

Altogether, these results allow us to propose an assembly model for tube formation, in which incipient singlelayer nanosheets of R6P6/P6E4 scroll along different crystallographic axes upon co-assembly at basic $\mathrm{pH}$. Scrolling of the collagen 2D lattice along the "a" axis, or $[1,0]$ plane, yields R6T_thin tubes, in which repeat spacings of $18.5 \AA$ are observed between triple helices oriented in lay- ers along the tube axis (Figure 5f). The non-perpendicular value of the tilt angle, $\Phi$, suggests that the collagen triple helices are inclined along the " $b$ " direction, which we propose is similar to the triple helix tilt angle, $\Omega$ (Figure $\mathbf{3 h}$ ), inferred from the cryo-EM images of the nanosheets (vide supra). Although we cannot conclusively state the degree to which the triple helices are tilted in the nanosheets, the data suggests that $\Omega=\Phi_{\text {R6T }_{\text {t thin }}}=82^{\circ}$. In a similar fashion, R6T_thick tubes result from corner to corner scrolling of

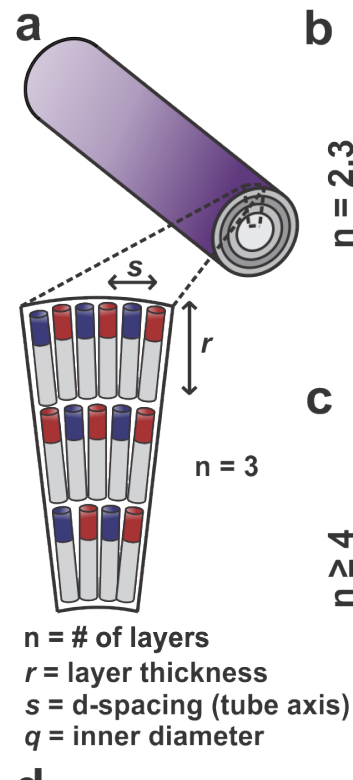

d

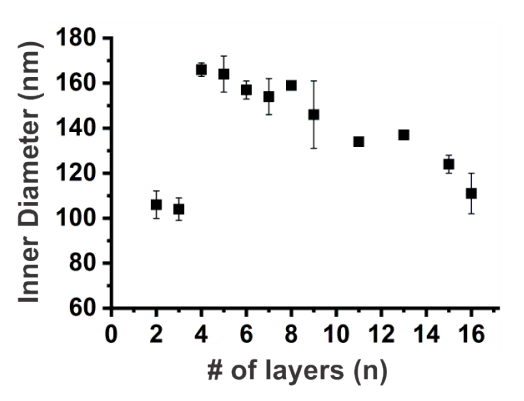

e

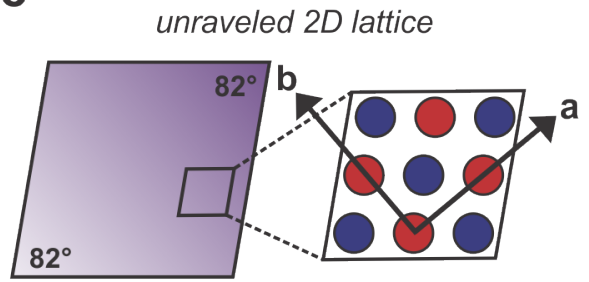

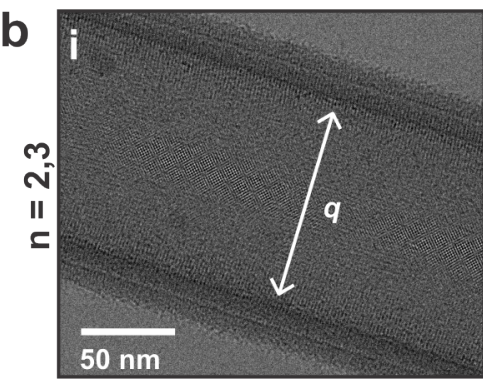
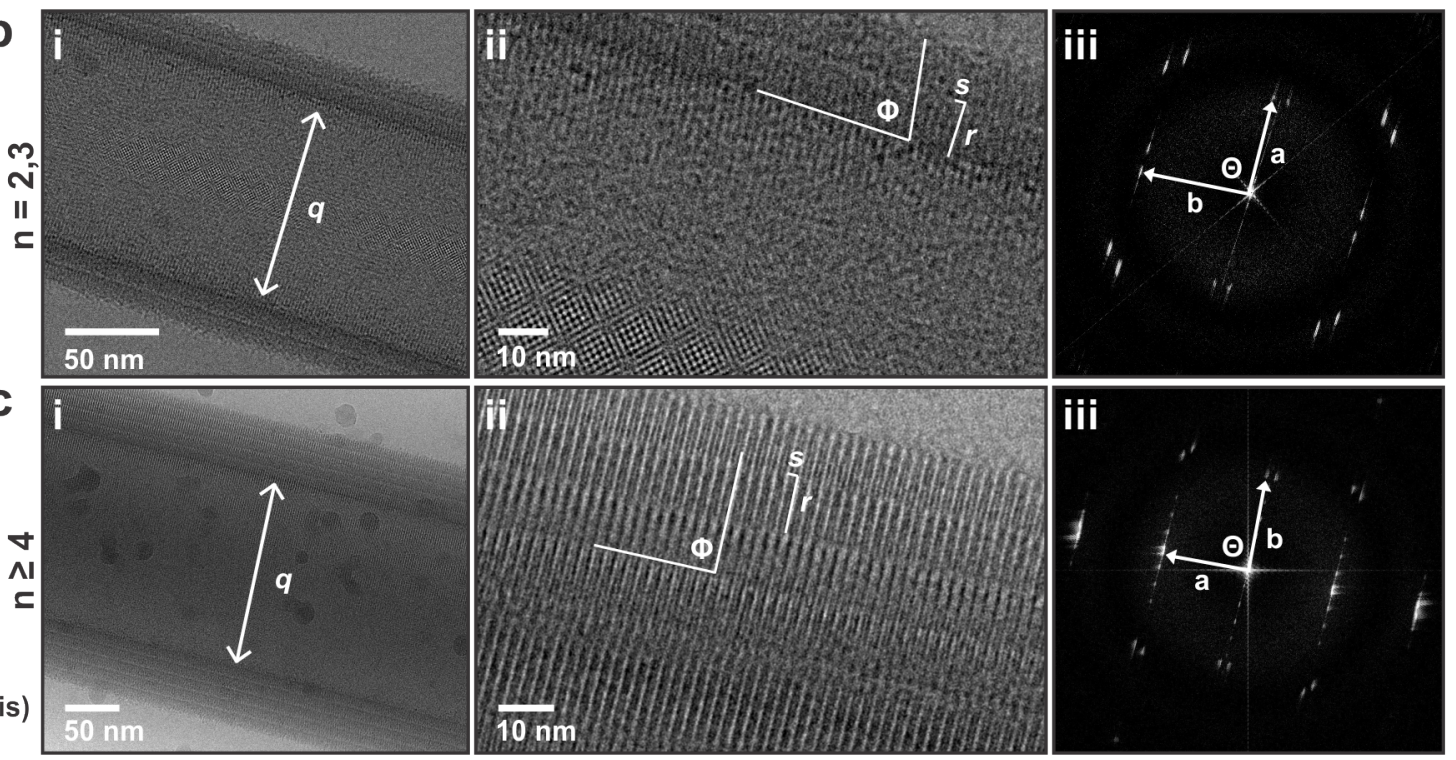

f R6T_thin $(n=2,3)$
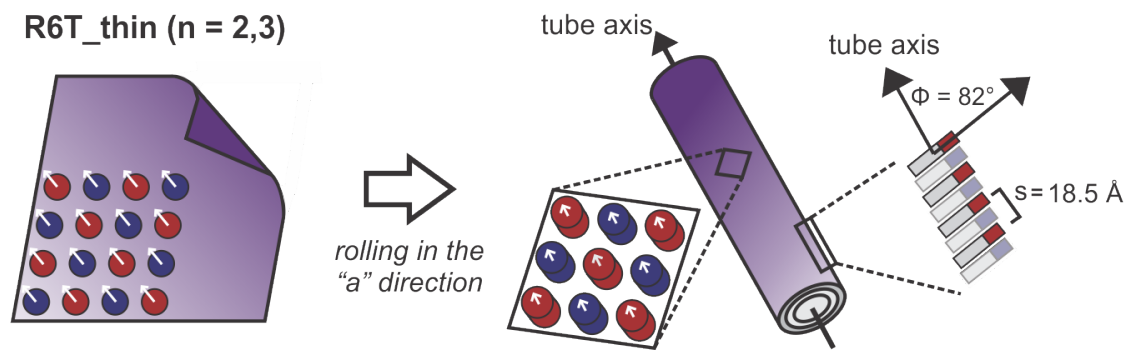

g R6T_thick $(n \geq 4)$

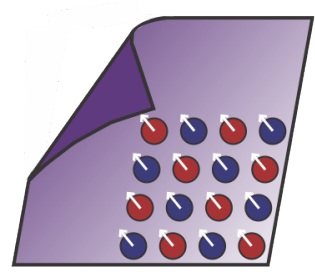

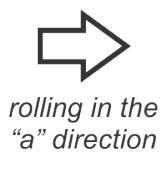

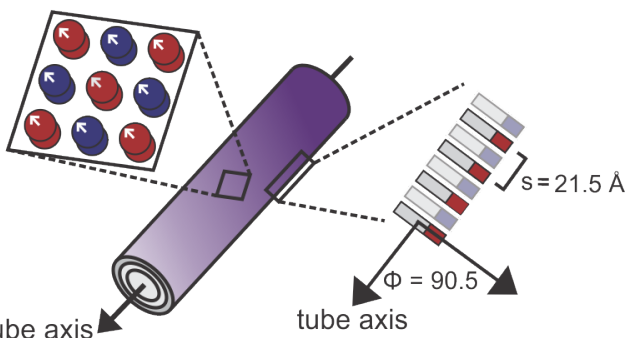

Figure 5. (a) Illustration of the proposed model for the collagen nanotubes depicting the various structural parameters of the tube assembly. High-resolution cryo-EM micrographs (i and ii) and corresponding FFT analysis (iii) of (b) R6T_thin and (c) R6T_thick tubes. (d) Inner diameter, $q$, as a function of the number of layers, n, comprising the tubes. (e) Packing model of collagen triple helical layers within the tube walls in which structurally adjacent heteromeric pairs of triple helices pack in an antiparallel orientation. Two lattice planes, labeled "a" and "b" define the [0,1] and [1,0] planes, respectively. (f) Proposed model for assembly of R6T_thin tubes. 2D sheets are scrolled along the "a" axis. The arrows indicate the tilted direction of the triple helices with respect to the surface. Scrolling along the "a" axis yields tubes with repeated distances of $18.5 \AA$ and $\Phi$ of $82^{\circ}$, which is consistent with highresolution cryo-EM micrographs. (g) Proposed model for assembly of R6T_thick tubes. 2D sheets are scrolled along the "b" axis. The arrows indicate the tilted direction of the triple helices with respect to the surface. Scrolling along the " $b$ " axis yields tubes with repeated distances of $21.5 \AA$ and $\Phi$ of $90.5^{\circ}$, which is consistent with high-resolution cryo-EM micrographs. 
nanosheets along the "b" axis, or [0,1] plane (Figure 5g), which leads to repeat distances of $21.5 \AA$ between triple helices within layers oriented along the tube axis. The tri- ple helices appear to be nearly perpendicular to the tube axis $\left(\Phi=90.5^{\circ}\right)$, which would only be possible if the tilting occurred out of the plane of the lamellar cross-section

Table 1. Selected Structural Parameters from Cryo-EM Analysis

\begin{tabular}{|c|c|c|c|c|c|c|c|c|}
\hline Species & layers $(\mathrm{n})$ & count $(\mathrm{N})$ & $\mathrm{a}(\AA)$ & $\mathrm{b}(\AA)$ & $\Phi\left({ }^{\circ}\right)$ & $\Theta\left({ }^{\circ}\right)$ & $s(\mathrm{~nm})$ & $q(\mathrm{~nm})$ \\
\hline R6P6/P6E4 NS & - & 10 & $21.6 \pm 0.1$ & $18.6 \pm 0.1$ & - & $90.6 \pm 0.4$ & - & - \\
\hline R6T_Thin & $\mathrm{n}=2,3$ & 14 & $21.5 \pm 0.1$ & $18.5 \pm 0.2$ & $82.2 \pm 0.2$ & $90.7 \pm 0.2$ & $18.5 \pm 0.0$ & $105.1 \pm 5.8$ \\
\hline R6T_Thick & $\mathrm{n} \geq 4$ & 31 & $21.5 \pm 0$ & $19.5 \pm 0.2$ & $89.9 \pm 0.2$ & $90.5 \pm 0.2$ & $21.5 \pm 0.1$ & $165-102$ \\
\hline
\end{tabular}

along the scrolling direction. These two scrolling mechanisms create tubes having distinctly different supramolecular architectures, even though they are based on closely related 2D unit cells. Moreover, the correspondence between the metrical parameters of the sheet and tube structures indicate that the unit cells of these two polymorphs are clearly related at a fundamental structural level. However, despite the similarity in 2D unit cells between the two populations of tubes, the degree of lamination depends strongly on the scrolling direction. The extent of scrolling is clearly limited along the crystallographic "a"direction, but can achieve high degrees of lamination (up to at least 16 layers) when scrolling along the " $b$ "direction.

We hypothesized that the preference for tube versus sheet formation in co-assemblies of R6P6/P6E4 was related to the degree of excess charge present at the assembly surface. At $\mathrm{pH}$ values in which one would expect a high net charge on the assembly, that is, under assembly conditions in which the buffer $\mathrm{pH}$ values diverges significantly from the estimated pI of the CMP mixture (Table S2), sheet formation was observed in solution (i.e., R6P6/P6E4 co-assembled under neutral or acidic conditions). In contrast, $\mathrm{pH}$ values that partially neutralized this excess surface charge yielded multi-lamellar tubes (i.e., R6P6/P6E4 and R4P6/P6E4 tubes grown under basic and neutral conditions, respectively). To verify this hypothesis, zeta potential measurements were conducted for all assemblies grown under the tested $\mathrm{pH}$ buffer conditions. The zeta potential values of R6P6/P6E4 assemblies decrease by 50 percent as the buffer $\mathrm{pH}$ increases from 6 to 8 (Figure 6a), which suggests that surface charge plays a critical role in determining the morphology of the final assembled structure. In conjunction with the slightly negative zeta potential of R4P6/P6E4 tubes at neutral $\mathrm{pH}$, these results suggest that tube formation occurs under conditions that reduce excess charge at the assembly surface (Figure 6a).

In addition to modulating the assembly $\mathrm{pH}$, the structural evolution of the peptide assemblies could be controlled through blending the two different positively charged CMPs at different compositional ratios. We hypothesized that this process could attenuate the level of excess positive surface charge at neutral $\mathrm{pH}$ and thereby result in a compositionally dependent morphological transition. To test this hypothesis, ternary peptide mixtures were prepared in MOPS buffer (20 mM, pH 7.0). The concentration of P6E4 was held constant while the compositional ratio of R4P6 versus R6P6 was continuously varied over the entire concentration range. As the relative concentration of R4P6 within the mixture increases, the zeta potential of the assembly solution decreases from ca. $+17 \mathrm{mV}$ to $-5 \mathrm{mV}$ (Figure 6b). TEM images at the various ratios confirm the morphological transition from sheets to tubes as a result of this variation in composition (Figure S22).

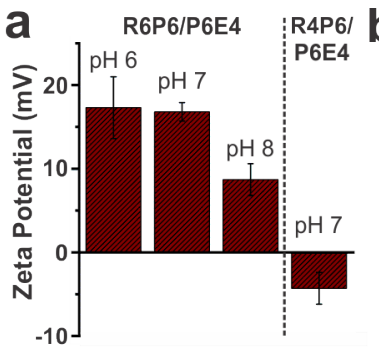

C

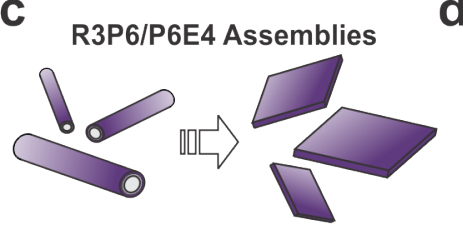

acidic $\mathrm{pH}$ neutral pH

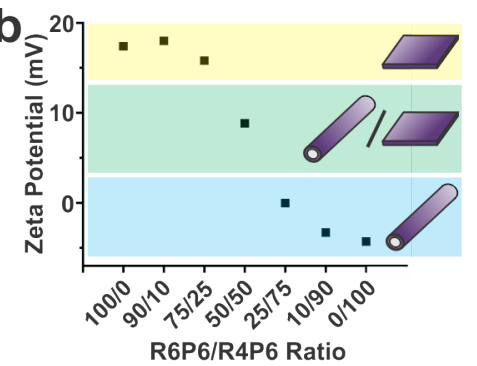
R6P6/R4P6 Ratio

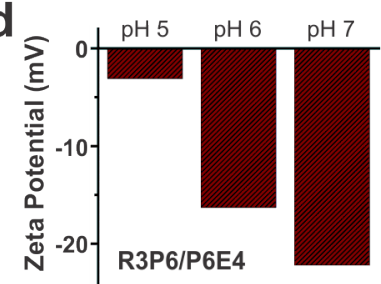

R3P6/P6E4

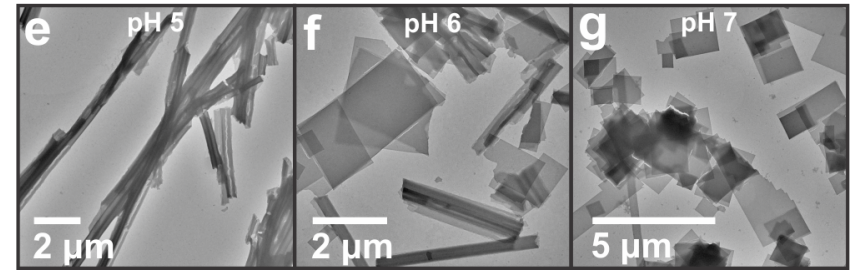

Figure 6. $\mathrm{pH}$ and zeta potential studies of peptide assemblies. (a) Zeta potential values of R6P6/P6E4 and R4P6/P6E4 assemblies $(0.2 \mathrm{mg} / \mathrm{mL})$ assembled under various $\mathrm{pH}$ conditions. (b) Zeta potential values of ternary peptide assemblies (grown in MOPS pH 7.0 buffer) as a function of R6P6/R4P6 ratio, while the concentration of P6E4 remains constant. (c) Scheme detailing the assembly conditions for R3P6/P6E4 sheets and tubes as a function of $\mathrm{pH}$. (d) Zeta potential values of R3P6/P6E4 assembled under various $\mathrm{pH}$ conditions. Stained TEM images of R3P6/P6E4 assembled in (e) acetate buffer (20 mM, pH 5.0), (f) MES buffer (20 mM, pH 6.0), and (g) MOPS buffer (20 mM, pH 7.0).

In a further test of the influence of surface charge, we anticipated that co-assembly of R3P6 and P6E4 triple helices would reverse the $\mathrm{pH}$ dependence of morphological transition observed for R6P6 and P6E4. The pI value for this mixed peptide system was estimated to be approximately 4.9 (Table S2), due to the extra negatively charged triad of P6E4 vis-à-vis R3P6. We hypothesize that co-assembly of the two peptides would result in tubes under acidic conditions, that is, in which the net surface charge is expected to 


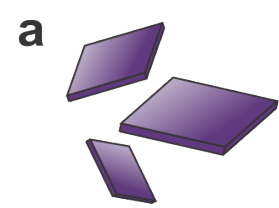

pH 7.0

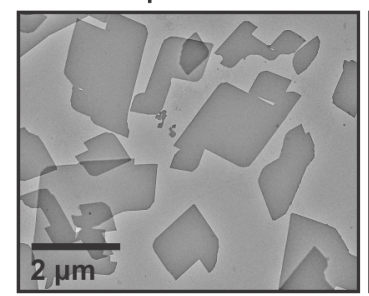

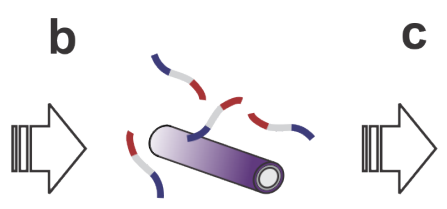

$\mathrm{pH} 8.5,1$ week

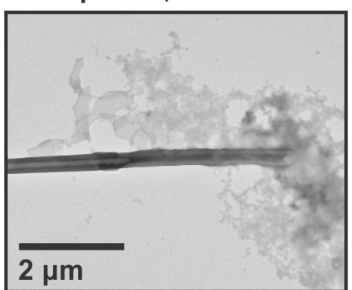

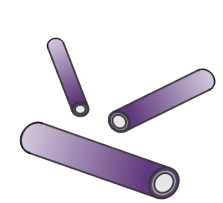

pH 8.5, 2 weeks

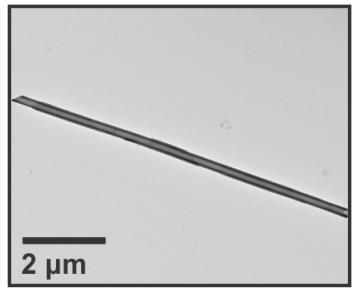

d

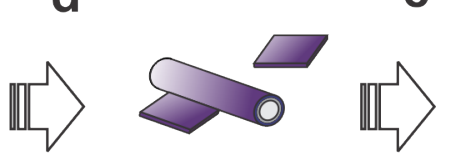

pH 7.0, 3 days

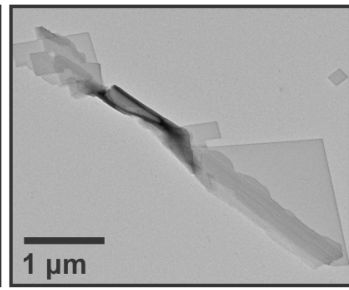

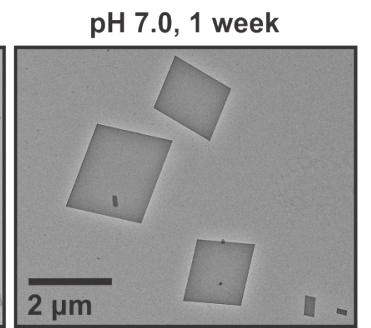

Figure 7. In situ interconversion between sheets and tube via $\mathrm{pH}$ modulation. Schematic representation of the inter-conversion (top) and the corresponding stained TEM images (bottom) of R6P6/P6E4 assemblies at various stages within the timepoint experiment: (a) R6P6/P6E4 nanosheet starting material assembled in MOPS buffer (20 mM, pH 7.0); (b) R6P6/P6E4 assemblies after 1 week of incubation in TAPS buffer (20 mM, pH 8.5); (c) R6P6/P6E4 tubes after 2 weeks of incubation in TAPS buffer (20 mM, pH 8.5); (d) R6P6/P6E4 assemblies after 3 days of incubation in MOPS buffer (pH 7.0); (e) R6P6/P6E4 nanosheets after 1 week of incubation in MOPS buffer ( $\mathrm{pH} 7.0)$.

be nearly neutral (Figure 6c). Zeta potential experiments confirm the increase in net negative charge associated with increasing the $\mathrm{pH}$ of the assembly solution (Figure 6d). As predicted, R3P6/P6E4 assembles into tubes under sufficiently acidic $\mathrm{pH}$ ( $\mathrm{pH} 5.0$; Figure 6e). As the buffer $\mathrm{pH}$ increases, a mixture of sheets and tubes are observed with a greater proportion of sheets at neutral pH (Figures 6f,g).

The change in morphology as a function of $\mathrm{pH}$, led us to investigate the potential for transforming the morphology of the mixed assemblies in situ. As a proof-of-concept, we employed the R6P6/P6E4 co-assembly system. Nanosheet solutions, prepared at neutral pH in MOPS buffer $(20 \mathrm{mM}$, $\mathrm{pH}$ 7.0), were sedimented via centrifugation and the supernatant was discarded. The nanosheets were resuspended in TAPS buffer (20 mM, pH 8.5) and the solution was incubated at ambient temperature. After two weeks, TEM images capture the successful transition from sheets to tubes (Figure 7a-c and S23-25). To demonstrate reversibility of this process, the tubes were sedimented and transferred to MOPS buffer (20 mM, pH 7.0) using the protocol described above. After incubation for 1 week at ambient temperature, the population of assemblies revert to nanosheets (Figure 7c-e and S26-27).

TEM images acquired during intermediate stages in this process reveal an asymmetry in the transition between these two structurally distinct polymorphs. When solutions of sheets were placed at basic $\mathrm{pH}$ values, the tubes did not appear to directly form from sheets, perhaps due to the symmetric bilayer structure. Instead, it seems that the sheets initially disassemble and then re-form into tubes. However, in the reverse direction, the TEM images provide evidence that the sheets emerge from tubes during the transition process (Figure $\mathbf{7 d}$ and S26). These results suggest that the reverted sheets originate from "unraveled or unscrolled" tubes. Furthermore, the sheets produced as a result of this pH-driven tube-to-sheet transformation exhibit a more uniform and well-defined morphology compared to the sheets assembled through the protocol employed with the previous experiments (Figure 7e and S27). In general, we have observed that the initial coassembly is a rapid process that is presumably under ki- netic control. In contrast, the pH-driven morphological transition occurs over a prolonged period that presumably permits the more controlled assembly of nanosheets. While the mechanism of this morphological transition remains to be elucidated in detail, these results nevertheless demonstrate that nanosheets and tubes can undergo dynamic interconversion.

Since we attribute the morphological transition between tubes and sheets to the effect of $\mathrm{pH}$ on the protonation/deprotonation state of the charged residues at the surface of the assembly, we reasoned that the ionic strength of the assembly buffer may also exert an influence due to charge screening of the electrostatic interactions between triple helical protomers. To address this, $\mathrm{NaCl}$ was added to a solution of pre-formed R6P6/P6E4 nanosheets ([peptide] $=1 \mathrm{mg} / \mathrm{mL}$ ) assembled in MOPs buffer (20 mM, pH 7.0). After several days, the sheet assemblies remained intact, even at concentrations up to 100 $\mathrm{mM} \mathrm{NaCl}$, indicating that charge screening is not sufficient to induce a commensurate morphological transition between sheets and tubes (Figure S28a,b). Furthermore, the formation of R6P6/P6E4 sheets assembled in the presence of $\mathrm{NaCl}$ (100 mM in $20 \mathrm{mM}$ MOPS buffer, $\mathrm{pH}$ 7.0), provides further evidence of the limited role of ionic strength on the assembly process (Figure S28c).

Finally, in order to assess the practical utility of these materials for applications, we investigated the effect of surface modification on the morphological behavior of the assemblies. As a proof-of-concept, $N$-terminal-modified biotin R6P6 peptide (b-R6P6; Figure S29) was doped (5\%) into a stock solution of R6P6 (1 mg/mL). This solution was then used for co-assembly experiments with P6E4 to produce biotin-doped sheets or tubes. The resultant assemblies were then incubated with either streptavidin-tagged Cy3B or green fluorescent protein (GFP) (Figure 8a). After the removal of excess dye, fluorescence optical microscopy was employed to confirm successful functionalization of the 1D and 2D assemblies (Figure 8b-e). These results demonstrate that the sheets and tubes can be functionalized with exogenous substrates without disrupting the morphological preference. These results point to- 
wards the potential for using these materials as responsive scaffolds for a variety of applications.

\section{CONCLUSION:}

We demonstrate that charge-complementary heteromeric pairs of designed collagen triple helices can spontaneously assemble into asymmetric 2D nanostructures (Janus nanosheets), which, under appropriate conditions, scroll to form high aspect-ratio crystalline nanotubes. The morphological outcome of this process depends on the excess surface charge, which can be controlled through peptide sequence, composition, and environmental conditions. While uncommon, environmentally responsive structural transitions between sheet and tube morphology have been observed for natural ${ }^{54-56}$ and synthetic ${ }^{57-60}$ protein assemblies - although this process doesn't necessarily depend on surface asymmetry. In addition, peptide-based Janus nanosheets don't necessarily scroll into tubes even though surface asymmetry may be present. ${ }^{61}$

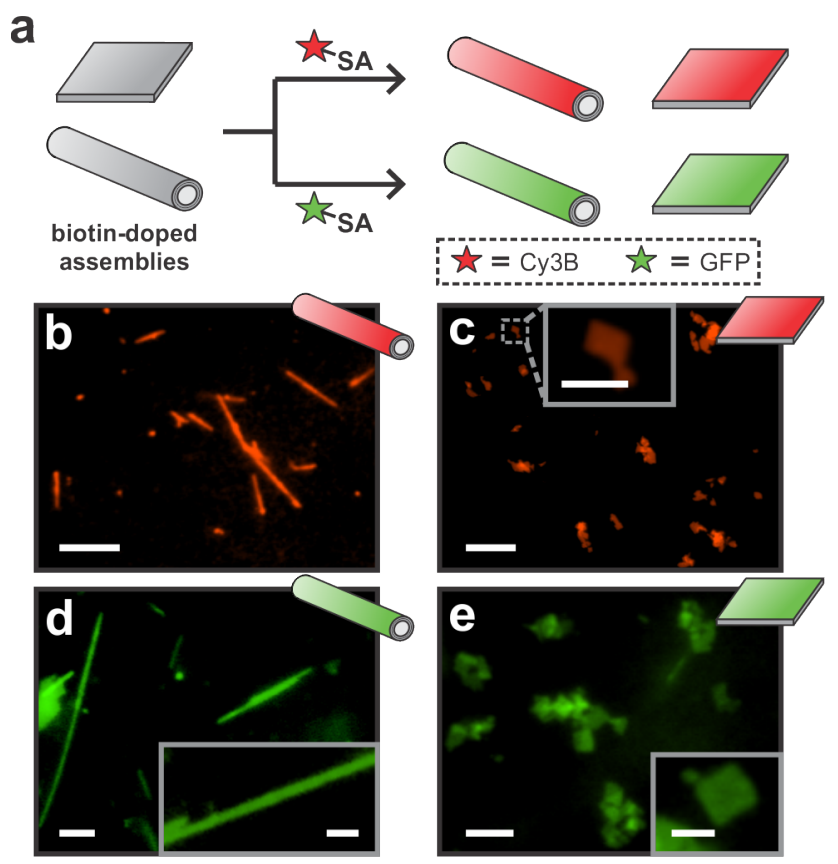

Figure 8. Surface functionalization of sheets and tubes. (a) Scheme detailing the attachment of SA-Cy3B and SA-GFP onto b-R6P6/P6E4 assemblies. Fluorescent optical micrographs of (b) b-R6P6/P6E4 tubes and (c) b-R6P6/P6E4 sheets functionalized with Сy3B; and (d) b-R6P6/P6E4 tubes and (e) bR6P6/P6E4 sheets functionalized with GFP. Scale bars $=4 \mu \mathrm{m}$ (inset scale bars $=2 \mu \mathrm{m}$ ).

The phenomenon of scrolling of 2D materials has been previously reported.62-70 In a majority of these cases, unbalanced surface stresses arise from asymmetric surface structures, which results in scrolling of sheets into tubular assemblies. We propose that a similar mechanism operates within these heteromeric collagen assemblies. However, in this work, the surface stresses and, consequently, the morphological transition, can be modulated through the development of differential surface charge between two structurally distinct interfaces. The two-component peptide system that we have developed may represent a more flex- ible platform to control this morphological transition since the sequences of the two peptides can be independently designed in order to enhance or abrogate this process (vide supra). This work highlights the potential for fabricating complex, transformable materials at the nanoscale, using simple biologically derived assembly motifs. We envision that these structures will provide new opportunities for creation of dynamically responsive materials that can be tailored for specific applications through incorporation of molecular mechanisms that can actuate this higher-order structural transitions.

\section{EXPERIMENTAL METHODS:}

Materials and Methods. Chemical reagents were purchased from Sigma Aldrich Chemical Co. (St. Louis, MO) and used without further purification. Fmoc-Gly-HMPTentaGel resins were purchased from Anaspec (Fremont, CA). Biotin-PEG2-acid was purchased from Broadpharm (San Diego, CA). Biotin-GFP was purchased from ProteinMods (Madison, WI). Peptides were quantified by mass and dissolved in water, followed by separation into aliquots of known amounts of peptides, and lyophilized. Matrix-assisted laser desorption ionization time-of-flight mass spectrometry (MALDI-TOF) data were collected using an Applied Biosystem 4700 mass spectrometer (positive reflector mode; accelerating voltage: $20 \mathrm{kV}$ ) and using $\alpha$-cyano-4-hydroxycinnamic acid (CHCA) as the ionization matrix.

Peptide Synthesis and Purification. All peptides were prepared using microwave-assisted synthesis on a CEM Liberty solid-phase peptide synthesizer and Fmoc-GlyHMP-Tentagel resin. In addition, P6E4 and R6P6 peptides were also purchased commercially from Synpeptide Company, Ltd. (Shanghai, China). Standard Fmoc protection chemistry was employed with coupling cycles based on HBTU/DIEA-mediated activation protocols and baseinduced deprotection (20\% piperidine in DMF) of the Fmoc group. For the biotinylated peptide (b-R6P6), biotinPEG2-acid (Broadpharm, San Diego, CA) was used to cap the N-terminus of the peptide while the peptide was still attached to the resin (no final deprotection step). After coupling, the DMF/resin mixture was filtered and rinsed with acetone and then air-dried. The crude peptides were cleaved for $3 \mathrm{~h}$ with a cleavage solution consisting of 92.5\% TFA/ 2.5\% $\mathrm{H}_{2} \mathrm{O} / 2.5 \%$ DODT / 2.5\% TIS (TFA = trifluoroacetic acid, DODT $=3,6$ - Dioxa-1,8-octane-dithiol, TIS = Triisopropylsilane). After filtration, crude peptide product in TFA was precipitated with cold $\mathrm{Et}_{2} \mathrm{O}$ and centrifuged at $4{ }^{\circ} \mathrm{C}$. The supernatants were discarded, and the pellets were dried under vacuum overnight.

Crude peptides were purified using a Shimadzu LC-20AP reverse-phase high performance liquid chromatography (HPLC) instrument equipped with a preparative scale C18 column. Peptides were eluted with a linear gradient of water-acetonitrile with $0.1 \%$ trifluoroacetic acid (TFA). The target fractions were collected and lyophilized. The lyophilized peptides were repurified via HPLC under the same protocol described above, and lyophilized. Doubly pure peptides were dialyzed against HPLC-grade $\mathrm{H}_{2} \mathrm{O}$ to remove residual TFA $($ MWCO $=2000 \mathrm{Da})$. The resulting peptide solutions were lyophilized and stored at $-30^{\circ} \mathrm{C}$. 
Peptide Assembly Experiments. All annealing experiments were conducted using a BioRad T-100 thermal cycler. The annealing protocol consisted of heating peptide solutions to $90^{\circ} \mathrm{C}$ for $15 \mathrm{~min}$, followed by slow-cooling to either $25^{\circ} \mathrm{C}$ or $4^{\circ} \mathrm{C}\left(0.5^{\circ} \mathrm{C} / 2.5 \mathrm{~min}\right.$ rate $)$.

Assembly experiments involved annealing $\left(90^{\circ} \mathrm{C}\right.$ to $\left.4^{\circ} \mathrm{C}\right)$ positively charged peptides (R4P6, R6P6, R3P6; 1 $\mathrm{mg} / \mathrm{mL}$ ) in appropriate buffers (acetate $\mathrm{pH} 5.0$, MES $\mathrm{pH}$ 6.0, MOPS pH 7.0, TAPS pH 8.0). After at least one week of incubation (to promote homotrimeric triple helix formation), P6E4 in corresponding buffer $(1 \mathrm{mg} / \mathrm{mL})$ was annealed from $90^{\circ} \mathrm{C}$ to $25^{\circ} \mathrm{C}$ and added directly to the arginine-containing peptide solution and the solution was vortexed immediately. Mixed peptide solutions were allowed to sit at room temperature. The solutions became cloudy after a few minutes, and a white precipitate was observed in solution within one day indicating the presence of assembled peptide.

Circular Dichroism Spectropolarimetry. CD measurements were conducted on a Jasco J-810 or J-1500 CD spectropolarimeter. Three spectra were recorded and averaged from $260 \mathrm{~nm}$ to $190 \mathrm{~nm}$ at a scanning rate of 100 $\mathrm{nm} / \mathrm{min}$ and a bandwidth of $2 \mathrm{~nm}$. CD melting experiments were performed in the temperature range from $5^{\circ} \mathrm{C}$ to $85^{\circ} \mathrm{C}$ at a heating rate of ca. $20^{\circ} \mathrm{C} /$ hour. The intensity of the CD signal at $224 \mathrm{~nm}$ was monitored as a function of temperature. Rpn values were determined by dividing the $a b-$ solute maxima and minima values at 224 and $198 \mathrm{~nm}$, respectively.

Transmission Electron Microscopy. TEM images were collected with a Hitachi H-7700 transmission electron microscope at an accelerating voltage of $80 \mathrm{kV}$. TEM specimens were prepared by briefly mixing $2.5 \mu \mathrm{L}$ of peptide nanosheet solution with $2.5 \mu \mathrm{L}$ of aqueous uranyl acetate stain solution (1\%) directly on a 200-mesh carbon-coated copper grid. After $30 \mathrm{~s}$, the excess liquid was wicked away and the grids were air-dried.

Dynamic Light Scattering. Zeta potential measurements were conducted using a NanoPlus DLS Particle Size Analyzer instrument (Particulate Systems, Norcross, GA). Immediately prior to data collection, samples $(1 \mathrm{mg} / \mathrm{mL})$ were diluted 5 -fold $(0.2 \mathrm{mg} / \mathrm{mL})$ with the corresponding buffer. The diluted assembly solutions $(200 \mu \mathrm{L})$ were then measured using a $200 \mu \mathrm{L}$ disposable zeta cuvette (Micromeritics). The mean zeta potential values were calculated by the NanoPlus software using the Smoluchowski approximation. Three replicates were measured for each sample.

Atomic Force Microscopy. AFM images were collected with an Asylum MFP-3D atomic force microscope using tapping-mode in air. Images were obtained using ultrasharp AFM tips with a force constant of $5 \mathrm{~N} / \mathrm{m}$ and resonance frequency of $150 \mathrm{kHz}$ (Budget Sensors, SHR-150). Images were collected with a scanning rate of $1 \mathrm{~Hz}$. Samples were washed by centrifuging the peptide assembly solutions $(1 \mathrm{mg} / \mathrm{mL})$ and removing the supernatant buffer, followed by resuspension in equivalent volume of HPLCgrade $\mathrm{H}_{2} \mathrm{O}$. The peptide assembly solutions were then dropcast $(20 \mu \mathrm{L})$ onto freshly cleaved mica. After $5 \mathrm{~min}$, the solution was wicked away and allowed to dry.
Scanning Electron Microscopy. SEM images were collected with a Topcon DS-130F field emission scanning electron microscope operating at an accelerating voltage of 10 $\mathrm{kV}$. SEM specimens were prepared by dropcasting $5 \mu \mathrm{L}$ of R6P6/P6E4 tube solution onto a 200-mesh carbon-coated copper grid. After $30 \mathrm{~s}$ the excess liquid was wicked away and the grids were air-dried. The samples were then sputter-coated (using a Denton Desktop II) with $\mathrm{Au} / \mathrm{Pd}$ alloy for $2 \min$ (ca. $6 \mathrm{~nm}$ ).

Small-Angle/Wide-Angle X-ray Scattering Measurements. Synchrotron SAXS/WAXS measurements were performed at the 12-ID-B beamline of the Advanced Photon Source at Argonne National Laboratory. The sample-todetector distances were set such that the overall scattering momentum transfer, $q$, range was achieved from 0.005 to $0.9 \AA-1$, where $q=4 \pi \sin \theta / \lambda, 2 \theta$ denoting the scattering angle and $\lambda$ the $X$-ray wavelength. The wavelength was set at $0.9322 \AA$ A during the measurements. Scattered X-ray intensities were measured using a Pilatus 2M (DECTRIS Ltd.) detector. Measurements were conducted on aqueous solutions of R6P6/P6E4 assemblies ( $4 \mathrm{mg} / \mathrm{mL}$ for sheet sample; $2 \mathrm{mg} / \mathrm{mL}$ for tube sample) in $20 \mathrm{mM}$ MOPS buffer ( $\mathrm{pH}$ 7.0) or 20 mM TAPS buffer ( $\mathrm{pH} 8.0$ ) for sheets and tubes, respectively. A quartz capillary flow cell $(1.5 \mathrm{~mm}$ diameter) was employed to prevent radiation damage. The 2D scattering images were converted to 1D scattering curves through azimuthal averaging after solid angle correction and then normalizing with the intensity of the transmitted $\mathrm{X}$-ray beam, using the software package at beamline 12-IDB. The 1D curves of the samples were averaged and subtracted with the background measured from the corresponding buffers.

Cryo-EM. The nanosheet and tube samples were shipped and stored at $4^{\circ} \mathrm{C}$. Hereafter it was briefly centrifuged to obtain a denser solution. Then 3-4 $\mu \mathrm{L}$ of the suspensions were pipetted onto a glow-discharged QuantiFoil grid 1.2/1.3 or Lacey grid (300 mesh). In case of the $\mathrm{pH} 8.0$ samples, $8 \mu \mathrm{L}$ of the sample was mixed with $31 \mu \mathrm{L}$ of buffer (90 mM Tris $\mathrm{pH} 8.0$ ) and $1 \mu \mathrm{L} 5 \mathrm{~nm}$ gold beads before applying the solution to the grid. Grids were blotted for $3 \mathrm{~s}$ with blot force 1 and plunge-frozen in liquid ethane using a Vitrobot with the environmental chamber set at $100 \%$ humidity and $4^{\circ} \mathrm{C}$. Data were acquired on a Titan Krios electron microscope at $300 \mathrm{keV}$ (Thermo Fisher) in nanoprobe mode, with a GIF Quantum LS Imaging filter $(20 \mathrm{eV}$ slit width) and a K2 Summit electron counting direct detection camera (Gatan).

Datasets at zero tilt were collected at a microscope magnification of $130 \mathrm{k}$, after correction resulting in a calibrated pixel size $1.05 \AA$ and $47.6 \mathrm{k}$ magnification. The defocus was set in a range of $0.7-2.0 \mu \mathrm{m}$. The data were collected with a modified in-house script using SerialEM. ${ }^{71}$ Every position was selected manually by observation of crystal-like features and each of these positions was exposed by recording a $10 \mathrm{~s}$ movie with 50 frames totaling $\sim 63 \mathrm{e}-/ \AA^{2}$.

The Focus software package was used to dose weight and drift correct the images by Motioncor2.72-73 The images were selected on minimal drift and non-stacked sheets and complete tubes (in case of pH 8.0 samples). These images were analyzed with FIJI software package. ${ }^{74}$ Sheets: an FFT was obtained and average (Table 1). To identify the crys- 
tal habit, images with clear edges were selected and the angle between the measured unit cell in the FFT was compared with the averaged edge of the crystal. Tubes: images were selected showing complete tubes. The measurements of the inner diameter of the tubes, layer thickness (for tubes where $n<4$ ), and angle of the peptides within the layer were performed on the real images. The Moiré pattern lattice points were identified by measuring the FFT in the core of the tubes. An FFT on the layers was used to determine the spacing and order of the collagen triple helices in the direction of the tube. For tubes with layers $n>3$, the FFT was used to measure the layer thickness.

The images used in Figures 3d and $\mathbf{S 1 5 b}$ were enhanced by a filter, based on a Wiener Filter approach. ${ }^{75}$ To enhance lattice signal, images were filtered using an in-house algorithm, in which the filtered images were used as an overlay with $50 \%$ transparency on top of the original non-noise filtered image (Figures 3d and S15a) using the standard overlay feature in FIJI. Enhancing the signal in this way allows for better identification of crystal features like edges, lattice stress or other defects. ${ }^{42}$ Except for using the filter as an overlay, nothing was changed from the original filtering method, mathematical procedures and effects. These are extensively described in detail in Ref. 75.

Fluorescence Optical Microscopy. Imaging was accomplished on a Nikon Eclipse Ti microscope, operated by Nikon Elements software, a 1.49 NA CFI Apo 100x objective, perfect focus system. An Andor iXon Ultra 897 electron multiplying charge coupled device was used for image acquisition. Widefield epifluorescence illumination was provided by an X-Cite 120 lamp (Excelitas). All of the reported experiments were performed using the Quad Cube (cat. \#97327), TRITC (cat. \#96321, Chroma) and reflection interference contrast microscopy (RICM) (cat. \#97270, Nikon).

Experiments were conducted using no. 1.5 glass slides (25 $\times 75 \mathrm{~mm})$ that were cleaned by sonication in MilliQ $(18.2 \mathrm{M} \Omega / \mathrm{cm})$ water for $15 \mathrm{~min}$, followed by a second sonication in 200 proof ethanol for $15 \mathrm{~min}$, and then dried under a stream of $\mathrm{N}_{2}$. The slides were etched by piranha solution $(\mathrm{v} / \mathrm{v}=3: 7$ hydrogen peroxide/sulfuric acid please take caution as piranha is extremely corrosive and may explode if exposed to organics) for $30 \mathrm{~min}$ to remove residual organic material. The clean glass slides were mounted to a custom-made 30 well microfluidic chamber fabricated in-house from Delrin (McMasterCarr, cat. \# 8573K15). The nanosheet and tube solutions were added $(10 \mu \mathrm{L})$ to the wells and were immediately subjected to a was $(3 \times 100 \mu \mathrm{L})$ with $20 \mathrm{mM}$ MOPS or TAPS buffer (pH 7.0 or 8.0, respectively). Assembly location was determined using RICM imaging. After focusing using RICM, one widefield epifluorescence micrograph was acquired.

\section{ASSOCIATED CONTENT}

\section{Supporting Information}

MALDI-TOF MS, HPLC, TEM, CD, Cryo-EM data.

The Supporting Information is available free of charge on the ACS Publications website.

\section{AUTHOR INFORMATION}

\section{Corresponding Author}

*vcontic@emory.edu

\section{Present Addresses}

Andrea D. Merg - Department of Chemistry and Chemical Biology, University of California, Merced

Gavin Touponse - School of Medicine, Stanford University

Author Contributions

\#A.D.M and G.T. contributed equally.

\section{ACKNOWLEDGMENT}

This work was supported by the NSF (CHE-1808509 and DMR-2003962, V.P.C.), and used resources of the Advanced Photon Source, a U.S. Department of Energy (DOE) Office of Science User Facility operated for the DOE Office of Science by Argonne National Laboratory under Contract No. DE-AC0206CH11357. Funding is acknowledged from the Swiss National Science Foundation project 31003A_17002 (T.B.B.) and 200021_165669 (T.B.B. and J.P.A.). This study was supported by the Robert P. Apkarian Integrated Electron Microscopy Facility. Stained TEM images were acquired on a Hitachi HT7700 $120 \mathrm{kV}$ TEM at Emory University, which was supported by the Georgia Clinical and Translational Science Alliance under NIH award number UL1TR002378. The content is solely the responsibility of the authors and does not necessarily reflect the official views of the National Institutes of Health. The circular dichroism spectropolarimeter was acquired through funding from an NSF grant (DBI-1726544). We thank Prof. Tianquan Lian and Prof. Henning Stahlberg for use of the atomic force microscope and cryo-EM facility, respectively.

\section{REFERENCES}

1. Wang, P.; Meyer, T. A.; Pan, V.; Dutta, P. K.; Ke, Y., The Beauty and Utility of DNA Origami. Chem 2017, 2 (3), 359-382.

2. Yuan, C.; Ji, W.; Xing, R.; Li, J.; Gazit, E.; Yan, X., Hierarchically oriented organization in supramolecular peptide crystals. Nat. Rev. Chem. 2019, 3 (10), 567-588.

3. Luo, Q.; Hou, C.; Bai, Y.; Wang, R.; Liu, J., Protein Assembly: Versatile Approaches to Construct Highly Ordered Nanostructures. Chem. Rev. 2016, 116 (22), 13571-13632.

4. Kuan, S. L.; Bergamini, F. R. G.; Weil, T., Functional protein nanostructures: a chemical toolbox. Chem. Soc. Rev. 2018, 47 (24), 9069-9105.

5. Egelman, E. H., Cryo-EM of bacterial pili and archaeal flagellar filaments. Curr. Opin. Struct. Biol. 2017, 46, 31-37.

6. Wang, F.; Burrage, A. M.; Postel, S.; Clark, R. E.; Orlova, A.; Sundberg, E. J.; Kearns, D. B.; Egelman, E. H., A structural model of flagellar filament switching across multiple bacterial species. Nat. Commun. 2017, 8 (1), 960.

7. Raymond, D. M.; Nilsson, B. L., Multicomponent peptide assemblies. Chem. Soc. Rev. 2018, 47 (10), 3659-3720.

8. Swanekamp, R. J.; DiMaio, J. T. M.; Bowerman, C. J.; Nilsson, B. L., Coassembly of Enantiomeric Amphipathic Peptides into Amyloid-Inspired Rippled $\beta$-Sheet Fibrils. J. Am. Chem. Soc. 2012, 134 (12), 5556-5559.

9. Silk, M. R.; Mohanty, B.; Sampson, J. B.; Scanlon, M. J.; Thompson, P. E.; Chalmers, D. K., Controlled Construction of Cyclic d / l Peptide Nanorods. Angew. Chem. Int. Ed. 2019, 58 (2), 596601.

10. Hudalla, G. A.; Sun, T.; Gasiorowski, J. Z.; Han, H.; Tian, Y. F.; Chong, A. S.; Collier, J. H., Gradated assembly of multiple proteins into supramolecular nanomaterials. Nat. Mater. 2014, 13 (8), 829836. 
11. Wong, K. M.; Wang, Y.; Seroski, D. T.; Larkin, G. E.; Mehta, A. K.; Hudalla, G. A.; Hall, C. K.; Paravastu, A. K., Molecular complementarity and structural heterogeneity within coassembled peptide $\beta$-sheet nanofibers. Nanoscale 2020, 12 (7), 4506-4518.

12. Khalily, M. A.; Bakan, G.; Kucukoz, B.; Topal, A. E.; Karatay, A.; Yaglioglu, H. G.; Dana, A.; Guler, M. O., Fabrication of Supramolecular n/p-Nanowires via Coassembly of Oppositely Charged Peptide-Chromophore Systems in Aqueous Media. ACS Nano 2017, 11 (7), 6881-6892.

13. Parmar, A. S.; James, J. K.; Grisham, D. R.; Pike, D. H.; Nanda, V., Dissecting Electrostatic Contributions to Folding and SelfAssembly Using Designed Multicomponent Peptide Systems. J. Am. Chem. Soc. 2016, 138 (13), 4362-4367.

14. Pal, V. K.; Jain, R.; Roy, S., Tuning the Supramolecular Structure and Function of Collagen Mimetic Ionic Complementary Peptides via Electrostatic Interactions. Langmuir 2020, 36 (4), 1003-1013.

15. Okesola, B. O.; Mata, A., Multicomponent self-assembly as a tool to harness new properties from peptides and proteins in material design. Chem. Soc. Rev. 2018, 47 (10), 3721-3736.

16. Lu, H. D.; Charati, M. B.; Kim, I. L.; Burdick, J. A., Injectable shear-thinning hydrogels engineered with a self-assembling Dock-and-Lock mechanism. Biomaterials 2012, 33 (7), 21452153.

17. Ramachandran, S.; Tseng, Y.; Yu, Y. B., Repeated Rapid Shear-Responsiveness of Peptide Hydrogels with Tunable Shear Modulus. Biomacromolecules 2005, 6 (3), 1316-1321.

18. Shigemitsu, H.; Fujisaku, T.; Tanaka, W.; Kubota, R.; Minami, S.; Urayama, K.; Hamachi, I., An adaptive supramolecular hydrogel comprising self-sorting double nanofibre networks. Nat. Nanotechnol. 2018, 13 (2), 165-172.

19. Li, S.; Mehta, A. K.; Sidorov, A. N.; Orlando, T. M.; Jiang, Z.; Anthony, N. R.; Lynn, D. G., Design of Asymmetric Peptide Bilayer Membranes. J. Am. Chem. Soc. 2016, 138 (10), 3579-3586.

20. Pandya, M. J.; Spooner, G. M.; Sunde, M.; Thorpe, J. R.; Rodger, A.; Woolfson, D. N., Sticky-End Assembly of a Designed Peptide Fiber Provides Insight into Protein Fibrillogenesis. Biochemistry 2000, 39 (30), 8728-8734.

21. Papapostolou, D.; Smith, A. M.; Atkins, E. D. T.; Oliver, S. J.; Ryadnov, M. G.; Serpell, L. C.; Woolfson, D. N., Engineering nanoscale order into a designed protein fiber. Proc. Natl. Acad. Sci. U.S.A. 2007, 104 (26), 10853-10858.

22. Sharp, T. H.; Bruning, M.; Mantell, J.; Sessions, R. B.; Thomson, A. R.; Zaccai, N. R.; Brady, R. L.; Verkade, P.; Woolfson, D. N., Cryo-transmission electron microscopy structure of a gigadalton peptide fiber of de novo design. Proc. Natl. Acad. Sci. U.S.A. 2012, 109 (33), 13266-13271.

23. Fletcher, J. M.; Harniman, R. L.; Barnes, F. R. H.; Boyle, A. L.; Collins, A.; Mantell, J.; Sharp, T. H.; Antognozzi, M.; Booth, P. J.; Linden, N.; Miles, M. J.; Sessions, R. B.; Verkade, P.; Woolfson, D. N., Self-Assembling Cages from Coiled-Coil Peptide Modules. Science 2013, 340 (6132), 595-599.

24. Epstein, I. R.; Xu, B., Reaction-diffusion processes at the nano- and microscales. Nat. Nanotechnol. 2016, 11 (4), 312-319.

25. Rothemund, P. W. K., Folding DNA to create nanoscale shapes and patterns. Nature 2006, 440 (7082), 297-302.

26. Zhang, Y.; Pan, V.; Li, X.; Yang, X.; Li, H.; Wang, P.; Ke, Y., Dynamic DNA Structures. Small 2019, 15 (26), 1900228.

27. Hentzen, N. B.; Smeenk, L. E. J.; Witek, J.; Riniker, S.; Wennemers, H., Cross-Linked Collagen Triple Helices by Oxime Ligation. J. Am. Chem. Soc. 2017, 139 (36), 12815-12820.

28. Hentzen, N. B.; Islami, V.; Köhler, M.; Zenobi, R.; Wennemers, H., A Lateral Salt Bridge for the Specific Assembly of an ABC-Type Collagen Heterotrimer. J. Am. Chem. Soc. 2020, 142 (5), 2208-2212.

29. Siebler, C.; Erdmann, R. S.; Wennemers, H., Switchable Proline Derivatives: Tuning the Conformational Stability of the
Collagen Triple Helix by pH Changes. Angew. Chem. Int. Ed. 2014, 53 (39), 10340-10344.

30. Tanrikulu, I. C.; Raines, R. T., Optimal Interstrand Bridges for Collagen-like Biomaterials. J. Am. Chem. Soc. 2014, 136 (39), 13490-13493.

31. Tanrikulu, I. C.; Forticaux, A.; Jin, S.; Raines, R. T., Peptide tessellation yields micrometre-scale collagen triple helices. Nat Chem. 2016, 8, 1008 .

32. Li, I. C.; Hulgan, S. A. H.; Walker, D. R.; Farndale, R. W.; Hartgerink, J. D.; Jalan, A. A., Covalent Capture of a Heterotrimeric Collagen Helix. Org. Lett. 2019, 21 (14), 5480-5484.

33. Jalan, A. A.; Hartgerink, J. D., Pairwise interactions in collagen and the design of heterotrimeric helices. Curr. Opin. Chem. Biol. 2013, 17 (6), 960-967.

34. O'Leary, L. E. R.; Fallas, J. A.; Hartgerink, J. D., Positive and Negative Design Leads to Compositional Control in AAB Collagen Heterotrimers. J. Am. Chem. Soc. 2011, 133 (14), 5432-5443.

35. Russell, L. E.; Fallas, J. A.; Hartgerink, J. D., Selective Assembly of a High Stability AAB Collagen Heterotrimer. J. Am. Chem. Soc. 2010, 132 (10), 3242-3243.

36. Gauba, V.; Hartgerink, J. D., Self-Assembled Heterotrimeric Collagen Triple Helices Directed through Electrostatic Interactions. J. Am. Chem. Soc. 2007, 129 (9), 2683-2690.

37. Kotch, F. W.; Raines, R. T., Self-assembly of synthetic collagen triple helices. Proc. Natl. Acad. Sci. U.S.A. 2006, 103 (9), 3028-3033.

38. O'Leary, L. E. R.; Fallas, J. A.; Bakota, E. L.; Kang, M. K.; Hartgerink, J. D., Multi-hierarchical self-assembly of a collagen mimetic peptide from triple helix to nanofibre and hydrogel. Nat. Chem. 2011, 3 (10), 821-828.

39. Blum, A. P.; Kammeyer, J. K.; Rush, A. M.; Callmann, C. E. Hahn, M. E.; Gianneschi, N. C., Stimuli-Responsive Nanomaterials for Biomedical Applications. J. Am. Chem. Soc. 2015, 137 (6), 2140-2154.

40. Merg, A. D.; Touponse, G.; van Genderen, E.; Zuo, X.; Bazrafshan, A.; Blum, T.; Hughes, S.; Salaita, K.; Abrahams, J. P.; Conticello, V. P., 2D Crystal Engineering of Nanosheets Assembled from Helical Peptide Building Blocks. Angew. Chem. Int. Ed. 2019, 58 (38), 13507-13512.

41. Jiang, T.; Vail, O. A.; Jiang, Z.; Zuo, X.; Conticello, V. P., Rational Design of Multilayer Collagen Nanosheets with Compositional and Structural Control. J. Am. Chem. Soc. 2015, 137 (24), 7793-7802.

42. Merg, A. D.; van Genderen, E.; Bazrafshan, A.; Su, H.; Zuo, X.; Touponse, G.; Blum, T. B.; Salaita, K.; Abrahams, J. P.; Conticello, V. P., Seeded Heteroepitaxial Growth of Crystallizable Collagen Triple Helices: Engineering Multifunctional Two-Dimensional Core-Shell Nanostructures. J. Am. Chem. Soc. 2019, 141 (51), 20107-20117.

43. Jiang, T.; Xu, C.; Liu, Y.; Liu, Z.; Wall, J. S.; Zuo, X.; Lian, T.; Salaita, K.; Ni, C.; Pochan, D.; Conticello, V. P., Structurally Defined Nanoscale Sheets from Self-Assembly of Collagen-Mimetic Peptides. J. Am. Chem. Soc. 2014, 136 (11), 4300-4308.

44. Jiang, T.; Xu, C.; Zuo, X.; Conticello, V. P., Structurally Homogeneous Nanosheets from Self-Assembly of a CollagenMimetic Peptide. Angew. Chem. Int. Ed. 2014, 53 (32), 8367-8371.

45. Berisio, R.; Vitagliano, L.; Mazzarella, L.; Zagari, A., Crystal structure of the collagen triple helix model [(Pro-Pro-Gly)10]3. Protein Sci. 2002, 11 (2), 262-270.

46. Okuyama, K.; Miyama, K.; Mizuno, K.; Bächinger, H. P., Crystal structure of (Gly-Pro-Hyp)9: Implications for the collagen molecular model. Biopolymers 2012, 97 (8), 607-616.

47. Hongo, C.; Noguchi, K.; Okuyama, K.; Tanaka, Y.; Nishino, N., Repetitive Interactions Observed in the Crystal Structure of a Collagen-Model Peptide, [(Pro-Pro-Gly)9]3. J. Biochem. 2005, 138 (2), 135-144.

48. Feng, Y.; Melacini, G.; Taulane, J. P.; Goodman, M., AcetylTerminated and Template-Assembled Collagen-Based Polypeptides Composed of Gly-Pro-Hyp Sequences. 2. Synthesis 
and Conformational Analysis by Circular Dichroism, Ultraviolet Absorbance, and Optical Rotation. J. Am. Chem. Soc. 1996, 118 (43), 10351-10358.

49. Yang, W.; Chan, V. C.; Kirkpatrick, A.; Ramshaw, J. A. M.; Brodsky, B., Gly-Pro-Arg Confers Stability Similar to Gly-Pro-Hyp in the Collagen Triple-helix of Host-Guest Peptides. J. Biol. Chem. 1997, 272 (46), 28837-28840.

50. Ramshaw, J. A. M.; Shah, N. K.; Brodsky, B., Gly-X-Y Tripeptide Frequencies in Collagen: A Context for Host-Guest Triple-Helical Peptides. J. Struct. Biol. 1998, 122 (1), 86-91.

51. Okuyama, K., Revisiting the Molecular Structure of Collagen. Connective Tissue Res. 2008, 49 (5), 299-310.

52. Okuyama, K.; Xu, X.; Iguchi, M.; Noguchi, K., Revision of collagen molecular structure. Peptide Sci. 2006, 84 (2), 181-191.

53. Stewart, M., Computer image processing of electron micrographs of biological structures with helical symmetry. $J$. Electron Microsc. Tech. 1988, 9 (4), 325-358.

54. Larsson, H.; Wallin, M.; Edström, A., Induction of a sheet polymer of tubulin by Zn2+. Exp. Cell Res. 1976, 100 (1), 104-110.

55. Wolf, S. G.; Mosser, G.; Downing, K. H., Tubulin Conformation in Zinc-Induced Sheets and Macrotubes. J. Struct. Biol. 1993, 111 (3), 190-199.

56. Nogales, E.; Wolf, S. G.; Downing, K. H., Structure of the $\alpha \beta$ tubulin dimer by electron crystallography. Nature 1998, 391 (6663), 199-203.

57. Brodin, J. D.; Ambroggio, X. I.; Tang, C.; Parent, K. N.; Baker, T. S.; Tezcan, F. A., Metal-directed, chemically tunable assembly of one-, two- and three-dimensional crystalline protein arrays. Nat. Chem. 2012, 4 (5), 375-382.

58. Brodin, J. D.; Carr, J. R.; Sontz, P. A.; Tezcan, F. A., Exceptionally stable, redox-active supramolecular protein assemblies with emergent properties. Proc. Natl. Acad. Sci. U.S.A. 2014, 111 (8), 2897-2902.

59. Xu, M.; Zeng, R.; Xiang, J.; Yan, Q., Shaping Protein Amphiphilic Assemblies via Allosteric Effect: From 1D Nanofilament to 2D Rectangular Nanosheet. J. Am. Chem. Soc. 2019, 141 (35), 13724-13728.

60. Kim, C.-J.; Park, J.-e.; Hu, X.; Albert, S. K.; Park, S.-J., PeptideDriven Shape Control of Low-Dimensional DNA Nanostructures. ACS Nano 2020, 14 (2), 2276-2284.

61. Lin, Y.; Thomas, M. R.; Gelmi, A.; Leonardo, V.; Pashuck, E. T.; Maynard, S. A.; Wang, Y.; Stevens, M. M., Self-Assembled 2D FreeStanding Janus Nanosheets with Single-Layer Thickness. J. Am. Chem. Soc. 2017, 139 (39), 13592-13595.

62. Xiong, H.; Chen, C.-K.; Lee, K.; Van Horn, R. M.; Liu, Z.; Ren, B.; Quirk, R. P.; Thomas, E. L.; Lotz, B.; Ho, R.-M.; Zhang, W.-B.; Cheng, S. Z. D., Scrolled Polymer Single Crystals Driven by Unbalanced Surface Stresses: Rational Design and Experimental Evidence. Macromolecules 2011, 44 (19), 7758-7766.

63. Agbolaghi, S.; Zenoozi, S.; Hosseini, Z.; Abbasi, F., Scrolled/Flat Crystalline Structures of Poly(3-hexylthiophene) and Poly(ethylene glycol) Block Copolymers Subsuming Unseeded Half-Ring-Like and Seeded Cubic, Epitaxial, and Fibrillar Crystals. Macromolecules 2016, 49 (24), 9531-9541.

64. Burks, G. R.; Qi, H.; Gleeson, S. E.; Mei, S.; Li, C. Y., Structure and Morphology of Poly(vinylidene fluoride) Nanoscrolls. ACS Macro Lett. 2018, 7 (1), 75-79.

65. Cai, W.; Li, C. Y.; Li, L.; Lotz, B.; Keating, M.; Marks, D., Submicrometer Scroll/Tubular Lamellar Crystals of Nylon 6,6. Adv. Mater. 2004, 16 (7), 600-605.

66. White, H. M.; Hosier, I. L.; Bassett, D. C., Cylindrical Lamellar Habits in Monodisperse Centrally Branched Alkanes. Macromolecules 2002, 35 (18), 6763-6765.
67. Kim, J.-H.; Bohra, M.; Singh, V.; Cassidy, C.; Sowwan, M., Smart Composite Nanosheets with Adaptive Optical Properties. ACS Appl. Mater. Interfaces 2014, 6 (16), 13339-13343.

68. Jones, C. D.; Kennedy, S. R.; Walker, M.; Yufit, D. S.; Steed, J. W., Scrolling of Supramolecular Lamellae in the Hierarchical SelfAssembly of Fibrous Gels. Chem 2017, 3 (4), 603-628.

69. Wang, Y.; Kim, Y.; Lee, M., Static and Dynamic Nanosheets from Selective Assembly of Geometric Macrocycle Isomers. Angew. Chem. Int. Ed. 2016, 55 (42), 13122-13126.

70. Choi, H.; Cho, K. J.; Seo, H.; Ahn, J.; Liu, J.; Lee, S. S.; Kim, H.; Feng, C.; Jung, J. H., Transfer and Dynamic Inversion of Coassembled Supramolecular Chirality through 2D-Sheet to Rolled-Up Tubular Structure. J. Am. Chem. Soc. 2017, 139 (49), 17711-17714.

71. Mastronarde, D. N., Automated electron microscope tomography using robust prediction of specimen movements. $J$. Struct. Biol. 2005, 152 (1), 36-51.

72. Biyani, N.; Righetto, R. D.; McLeod, R.; Caujolle-Bert, D.; Castano-Diez, D.; Goldie, K. N.; Stahlberg, H., Focus: The interface between data collection and data processing in cryo-EM. J. Struct. Biol. 2017, 198 (2), 124-133.

73. Zheng, S. Q.; Palovcak, E.; Armache, J.-P.; Verba, K. A.; Cheng, Y.; Agard, D. A., MotionCor2: anisotropic correction of beaminduced motion for improved cryo-electron microscopy. Nat. Methods 2017, 14, 331.

74. Schindelin, J.; Arganda-Carreras, I.; Frise, E.; Kaynig, V.; Longair, M.; Pietzsch, T.; Preibisch, S.; Rueden, C.; Saalfeld, S.; Schmid, B.; Tinevez, J.-Y.; White, D. J.; Hartenstein, V.; Eliceiri, K.; Tomancak, P.; Cardona, A., Fiji: an open-source platform for biological-image analysis. Nat. Methods 2012, 9 (7), 676-682.

75. van Genderen, E.; Li, Y.-W.; Nederlof, I.; Abrahams, J. P., Lattice filter for processing image data of three-dimensional protein nanocrystals. Acta Crystallogr. D 2016, 72 (1), 34-39. 


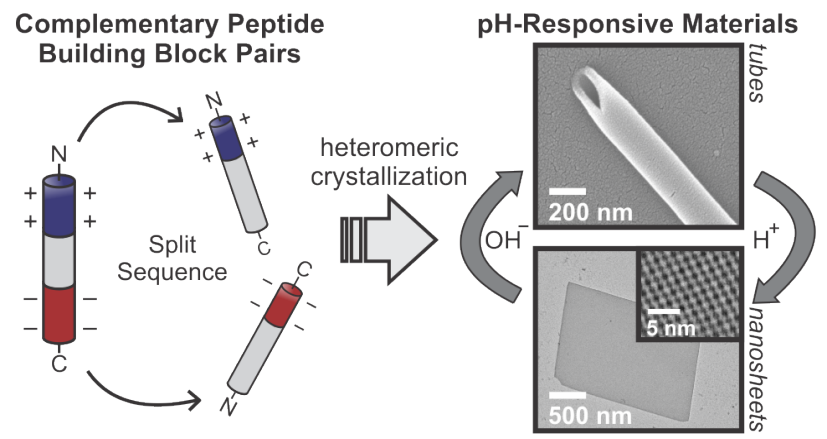




\section{Shape-Shifting Peptide Nanomaterials: Surface Asymmetry Enables pH- Dependent Formation and Interconversion of Collagen Tubes and Sheets}

Andrea D. Merg,,,$\uparrow$ Gavin Touponse, ${ }^{\#, \dagger}$ Eric van Genderen, ${ }^{\ddagger}$ Thorsten B. Blum, ${ }^{\ddagger}$ Xiaobing Zuo, ${ }^{\S}$ Alisina Bazrafshan,${ }^{\dagger}$ Hew Ming Helen Siaw,${ }^{\dagger}$ Arthur McCanna,${ }^{\perp}$ R. Brian Dyer, ${ }^{\dagger}$ Khalid Salaita,${ }^{\dagger}$ Jan Pieter Abrahams, ${ }^{\ddagger} \uparrow$ and Vincent P. Conticello ${ }^{*, \dagger}$

$\dagger$ Department of Chemistry, Emory University, Atlanta, Georgia 30322, United States

‡ Paul Scherrer Institut, CH-5232 Villigen Switzerland

$\S$ X-ray Science Division, Argonne National Laboratory, Lemont, Illinois 60439, United States

${ }^{\perp}$ The Robert P. Apkarian Integrated Electron Microscopy Core, Emory University, Atlanta, GA, 30322, United States

" Center for Cellular Imaging and NanoAnalytics, Biozentrum, University of Basel, CH-4058, Basel, Switzerland 


\section{Supporting Information}
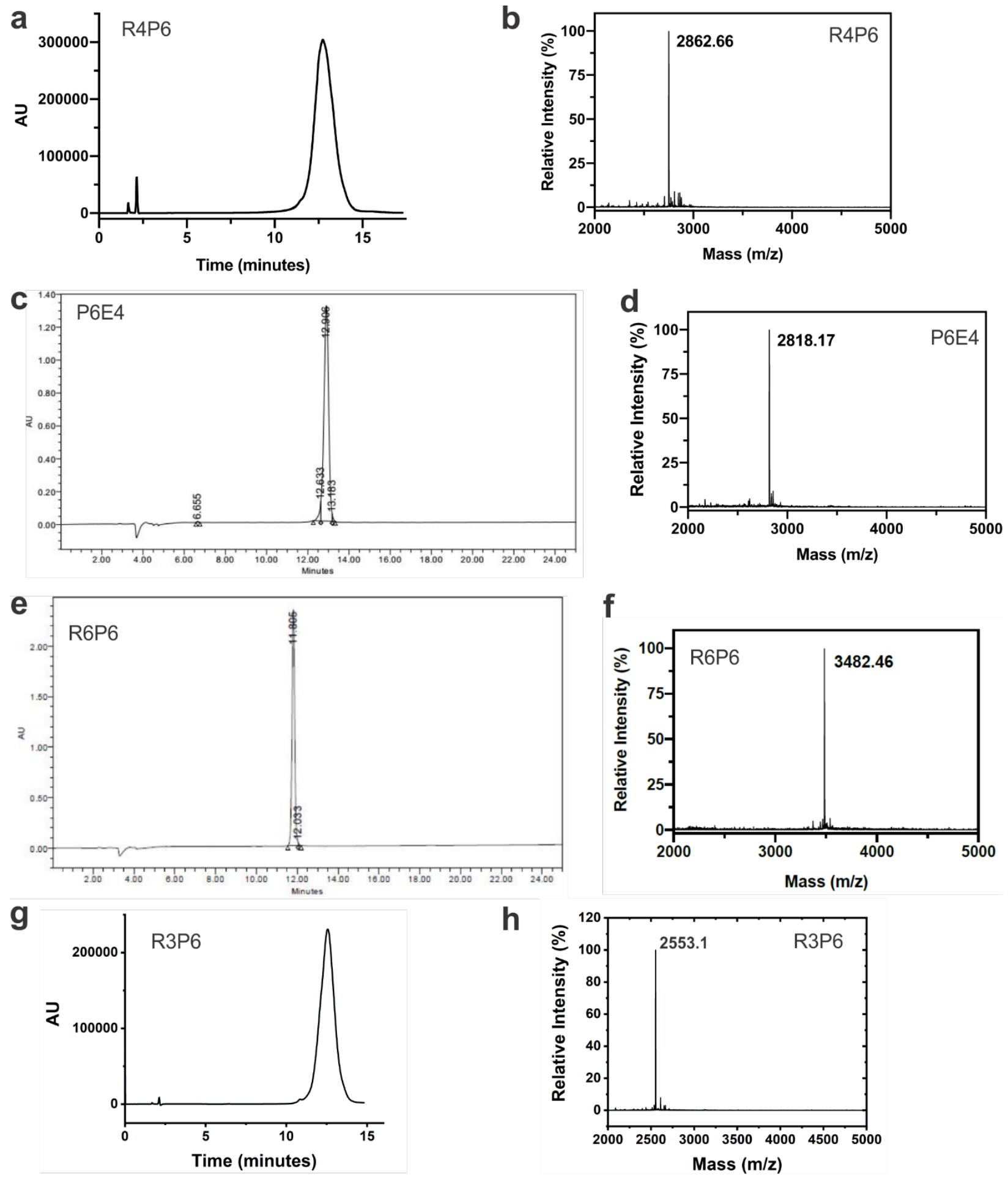

Figure S1. HPLC traces of (a) R4P6, (c) P6E4, (e) R6P6, and (g) R3P6. MALDI-TOF mass spectra of purified (b) R4P6, m/z $=2862.66\left(\mathrm{M}+\mathrm{H}^{+}\right)$; (d) P6E4, m/z $=2818.17\left(\mathrm{M}+\mathrm{H}^{+}\right)$; (f) R6P6, $\mathrm{m} / \mathrm{z}=3482.46\left(\mathrm{M}+\mathrm{H}^{+}\right)$; and (h) R3P6, $\mathrm{m} / \mathrm{z}=2553.1\left(\mathrm{M}+\mathrm{H}^{+}\right)$. 


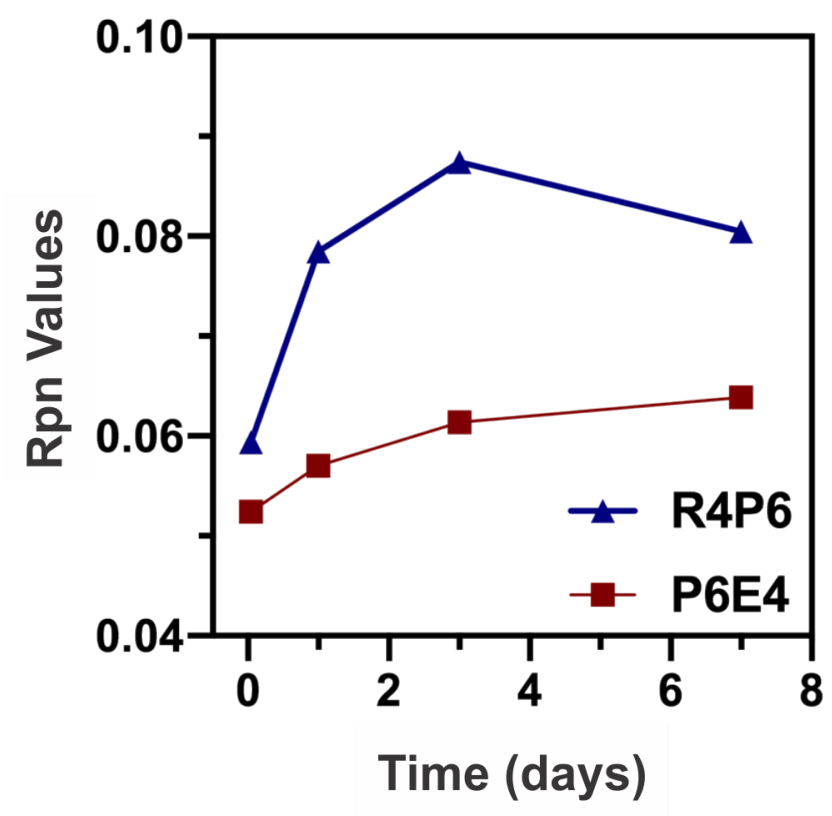

Figure S2. Rpn values of R4P6 and P6E4 in MOPS buffer (20 mM, pH 7.0) as a function of time.
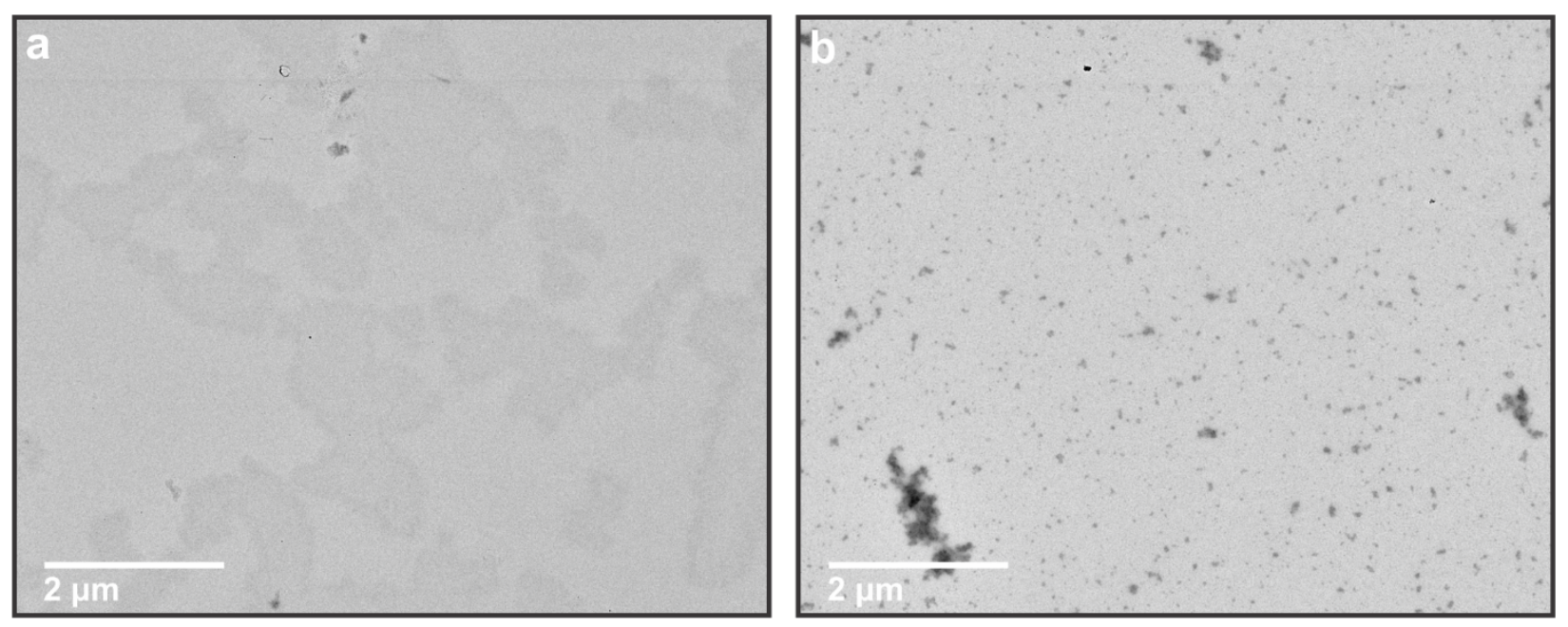

Figure S3. Stained TEM images of (a) R4P6 and (b) P6E4 solutions (1 mg/mL) after one week of incubation at ambient temperature in MOPS buffer (20 mM, pH 7.0). 

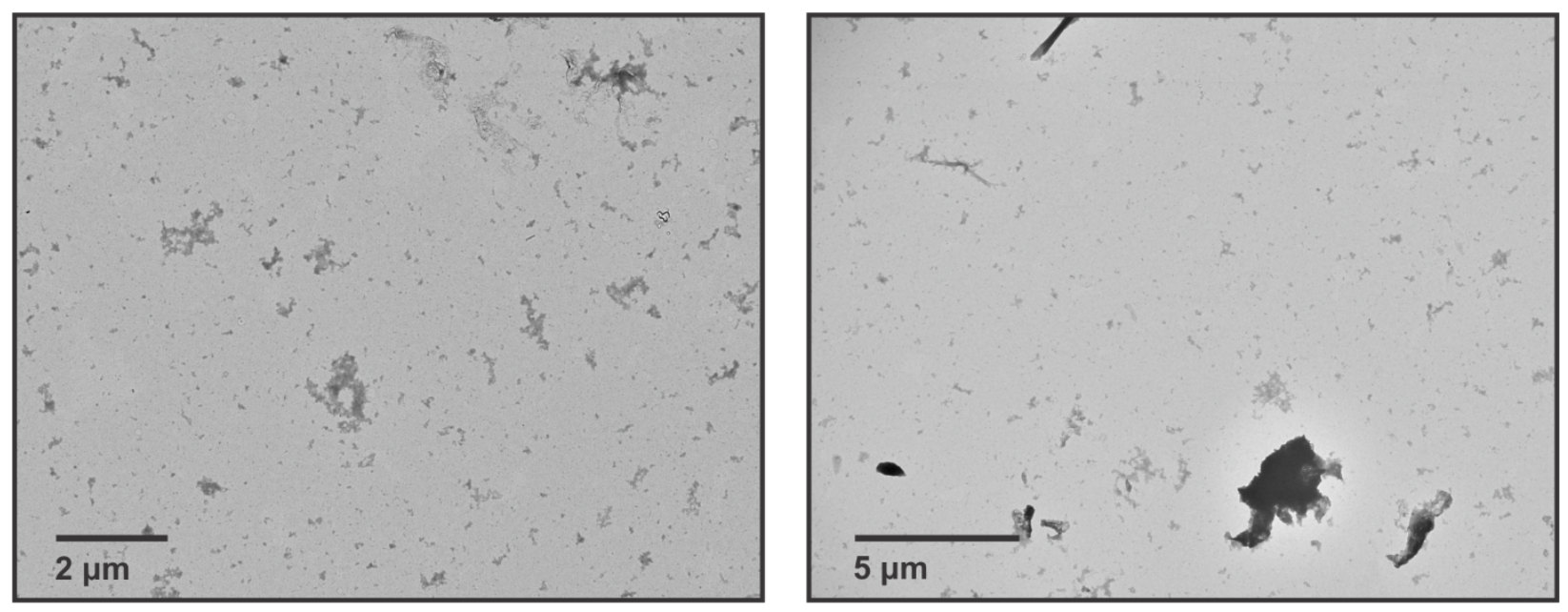

Figure S4. Stained TEM images of R4P6/P6E4 $(1 \mathrm{mg} / \mathrm{mL})$ after one week of incubation at ambient temperature in MOPS buffer $(20 \mathrm{mM}, \mathrm{pH} 7.0)$, using a one-pot annealing protocol. Illdefined aggregates were primarily observed.
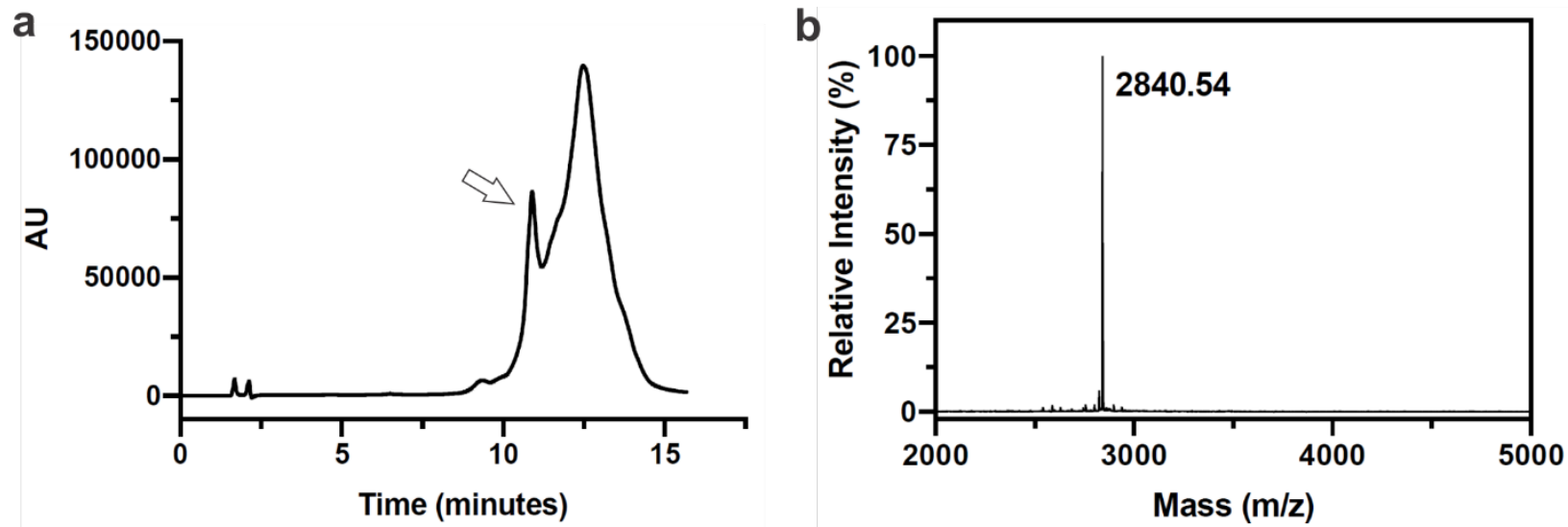

Figure S5. (a) HPLC trace of doubly purified CP262. (b) MALDI-TOF spectrum of purified CP262, $\mathrm{m} / \mathrm{z}=2840.54\left(\mathrm{M}+\mathrm{H}^{+}\right)$. Arrow in a signifies the peak containing free, unassociated peptide CP262. Peptide CP262 aggregates strongly to form higher order species in solution, which can be detected as the major component in the HPLC trace. Both free peptide and aggregated peptide display identical mass spectra. 

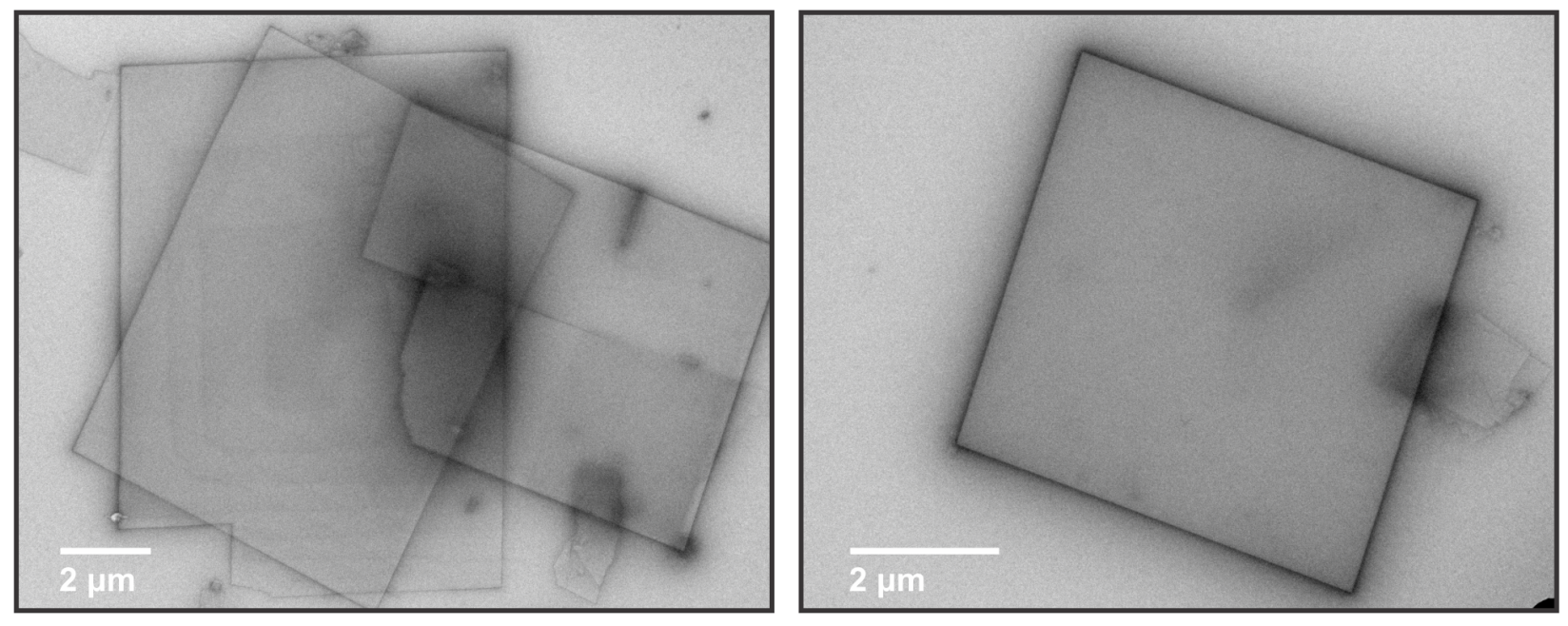

Figure S6. Stained TEM images of CP262 nanosheets $(1 \mathrm{mg} / \mathrm{mL})$ assembled in MOPS buffer (20 $\mathrm{mM}, \mathrm{pH} 7.0$ ) after 2 weeks of assembly time. 
a

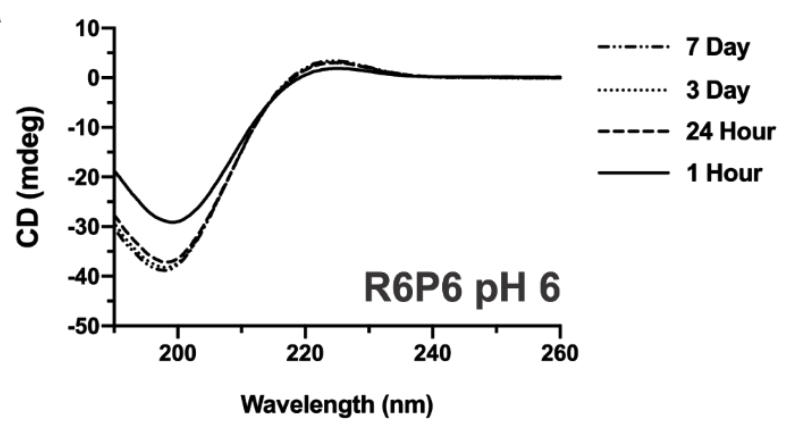

C

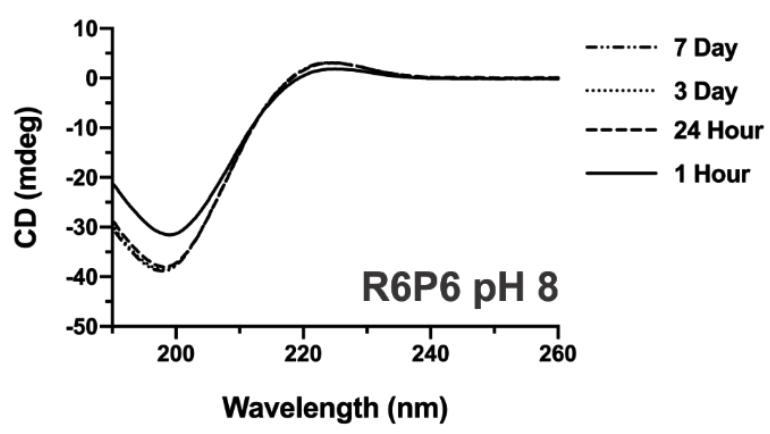

b

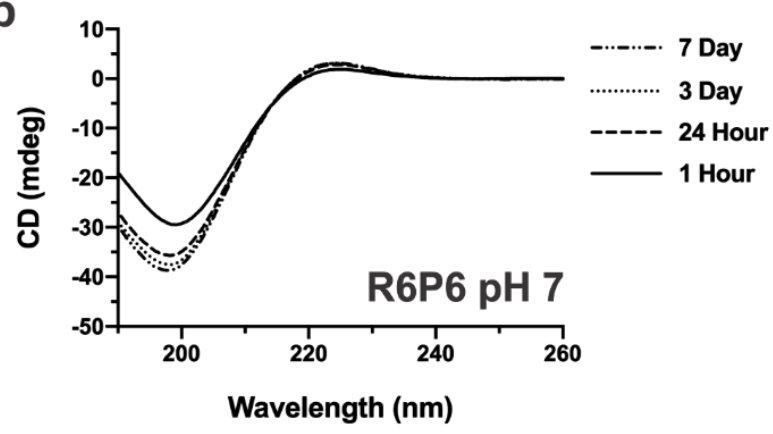

d

R6P6 Solutions: Rpn Values

\begin{tabular}{|c|c|c|c|c|}
\cline { 2 - 5 } \multicolumn{1}{c|}{} & $\begin{array}{c}\text { Rpn: } \\
\text { 1 hr. }\end{array}$ & $\begin{array}{c}\text { Rpn: } \\
\text { 1 day }\end{array}$ & $\begin{array}{c}\text { Rpn: } \\
\text { 3 days }\end{array}$ & $\begin{array}{c}\text { Rpn: } \\
\text { 1 week }\end{array}$ \\
\hline pH 6 & 0.065 & 0.081 & 0.082 & 0.087 \\
\hline pH 7 & 0.064 & 0.079 & 0.083 & 0.081 \\
\hline pH 8 & 0.059 & 0.080 & 0.081 & 0.081 \\
\hline
\end{tabular}

Figure S7. Circular dichroism (CD) spectra, collected at various timepoints, of R6P6 (1 mg/mL) in (a) MES buffer (20 mM, pH 6.0), (b) MOPS buffer (20 mM, pH 7.0), and (c) TAPS buffer (20 $\mathrm{mM}, \mathrm{pH}$ 8.0). (d) Table of Rpn values calculated from the CD spectra of a-c.
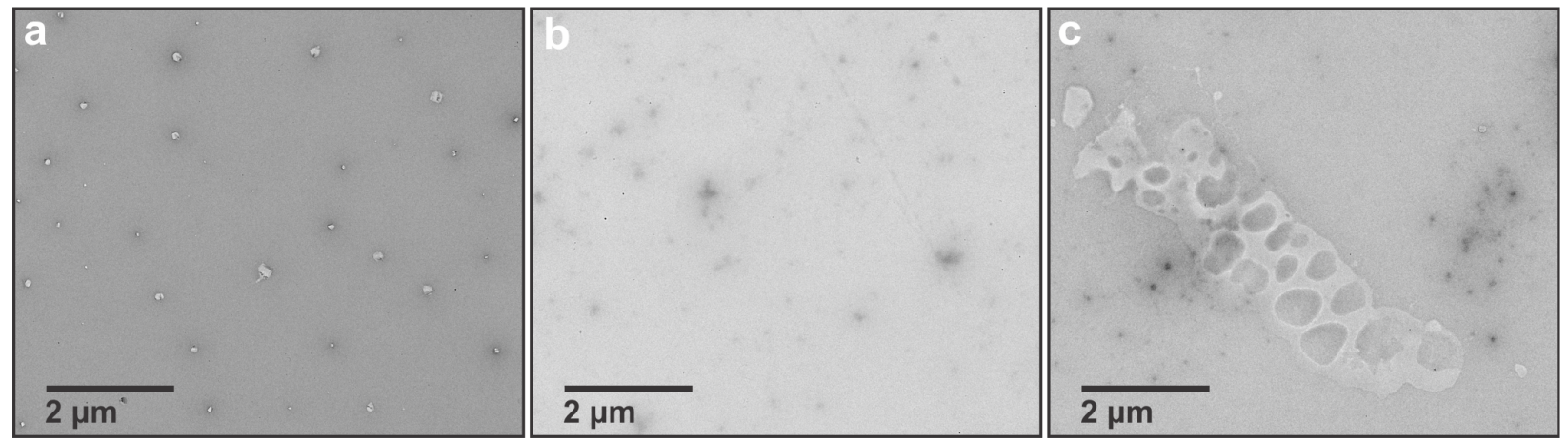

Figure S8. Stained TEM images, after one week of incubation at ambient temperature, of R6P6 in (a) MES buffer (20 mM, pH 6.0), (b) MOPS buffer (20 mM, pH 7.0), and (c) TAPS buffer (20 $\mathrm{mM}, \mathrm{pH}$ 8.0). No structurally defined assemblies were observed. 


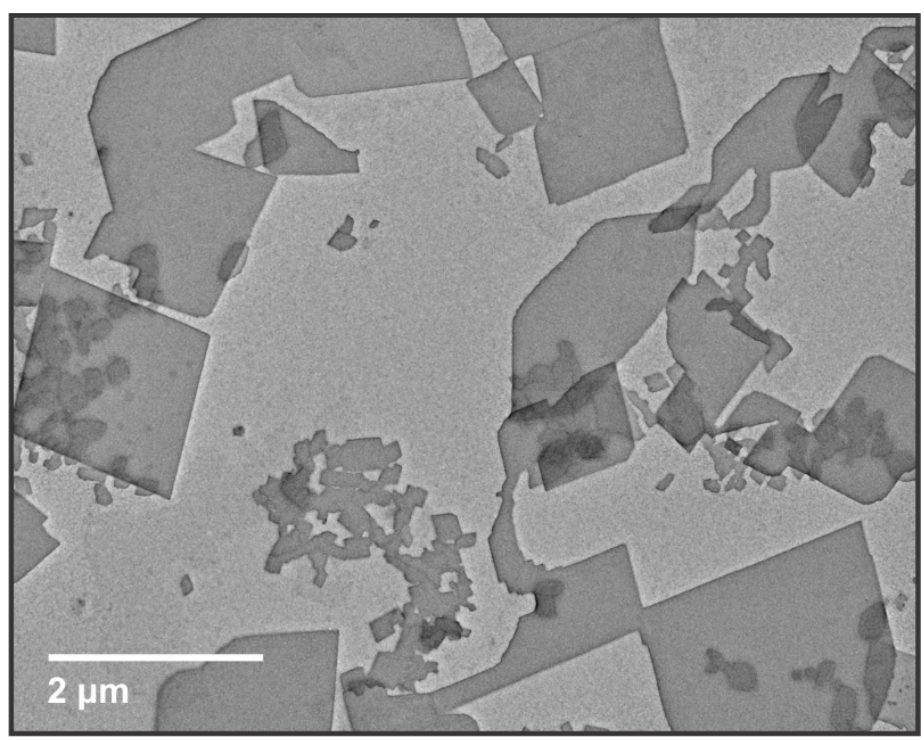

Figure S9. Stained TEM image of R6P6/P6E4 nanosheets $(1 \mathrm{mg} / \mathrm{mL})$ assembled in MES buffer (20 mM, pH 6.0) after one week of assembly time. 

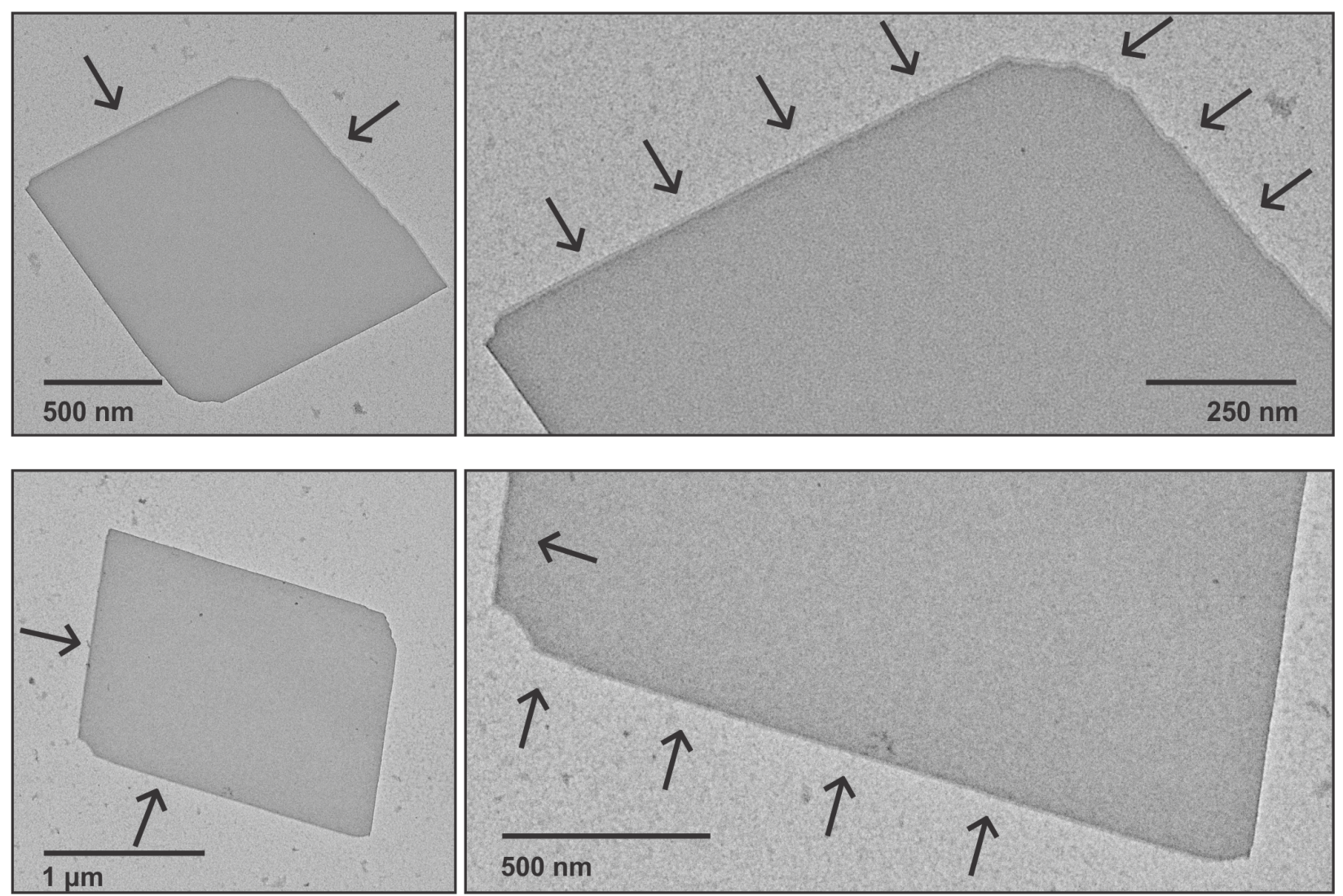

Figure S10. Stained TEM images of R6P6/P6E4 nanosheets $(1 \mathrm{mg} / \mathrm{mL})$ assembled in MOPS buffer $(20 \mathrm{mM}, \mathrm{pH} 7.0)$. Arrows indicate nanosheet periphery having different electron contrast, which suggests the presence of at least two separate layers. 


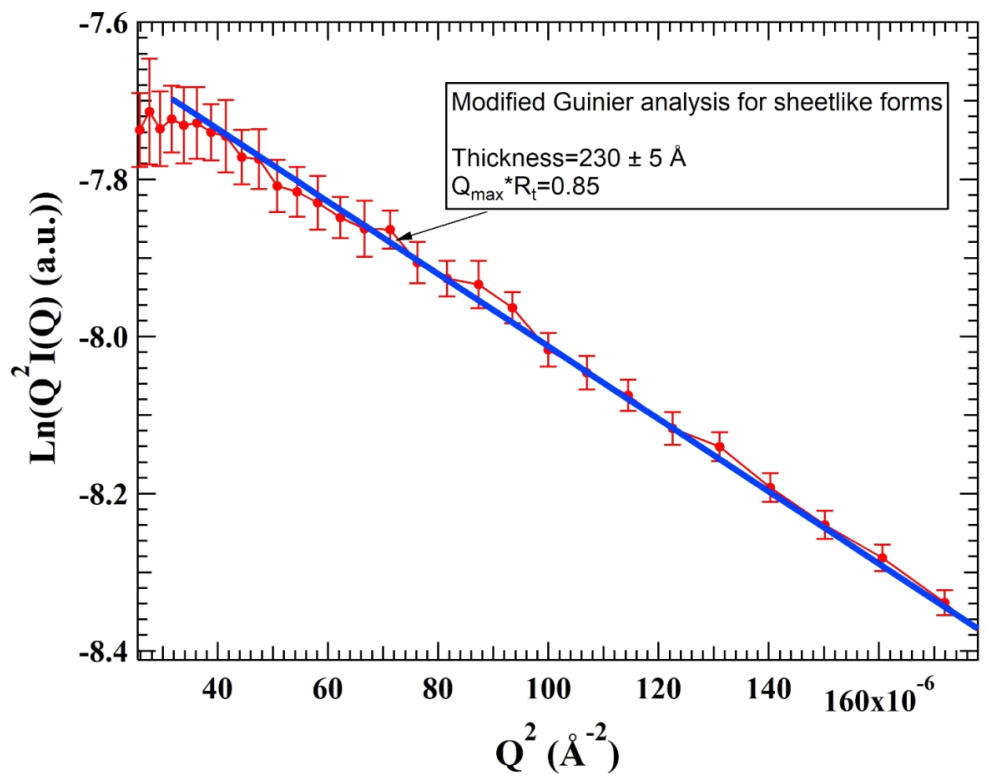

Figure S11. Guinier plot of experimental data for R6P6/P6E4 nanosheets (red) in MOPS buffer (20 mM, pH 7.0) fitted to an equation for sheet-like forms (blue line).

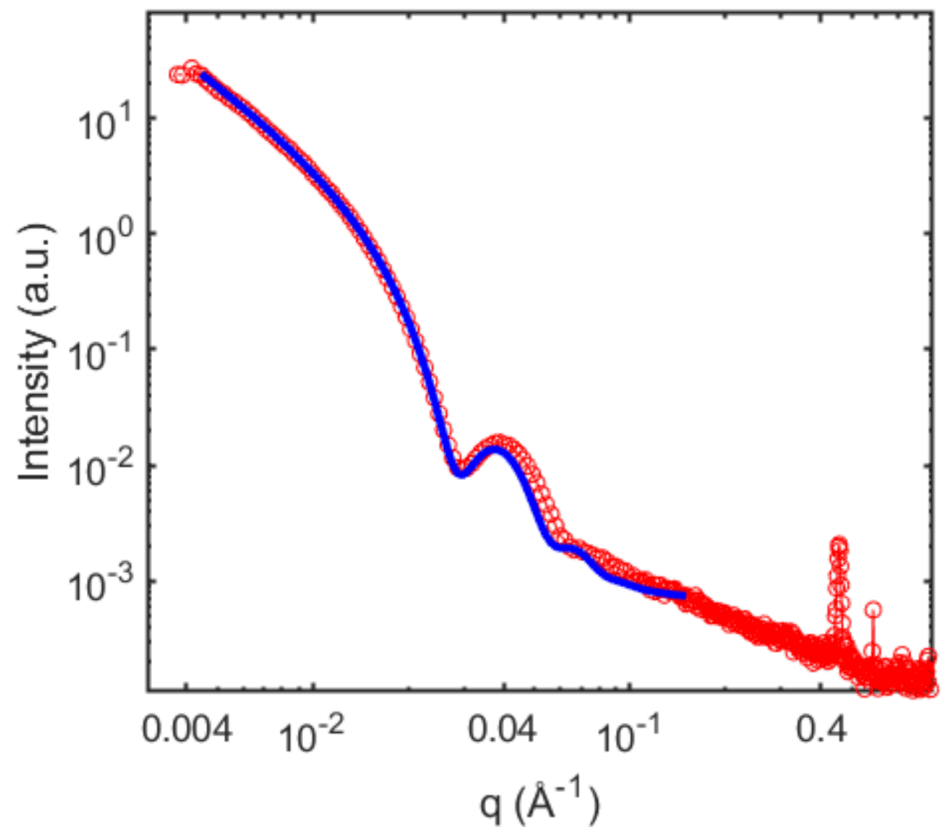

Figure S12. Direct fit of experimental SAXS scattering data (red) with lamella plot (blue) for R6P6/P6E4 nanosheets in MOPS buffer (20 mM, pH 7.0). 


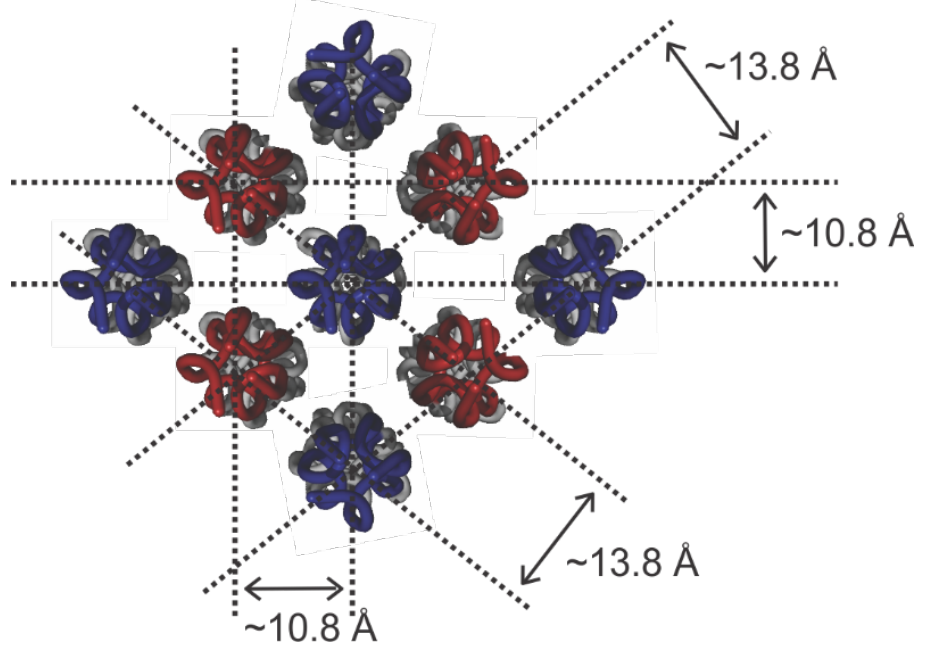

Figure S13. Proposed model of 2D lattice structure of R6P6/P6E4 nanosheets with $d$-spacings obtained from SAXS/WAXS scattering curves. The $d$-spacing associated with the $10.8 \AA$ distance corresponds to the $[2,0]$ plane, as it represents half the distance $(21.8 \AA / 2)$ associated with the $[1,0]$ plane as determined from the cryo-EM analysis. The $d$-spacing at $13.8 \AA$ represents a slightly contracted composite of the distances associated with $[1,1]$ and $[1,-1]$ planes, which cannot be resolved in the SAXS measurements. 


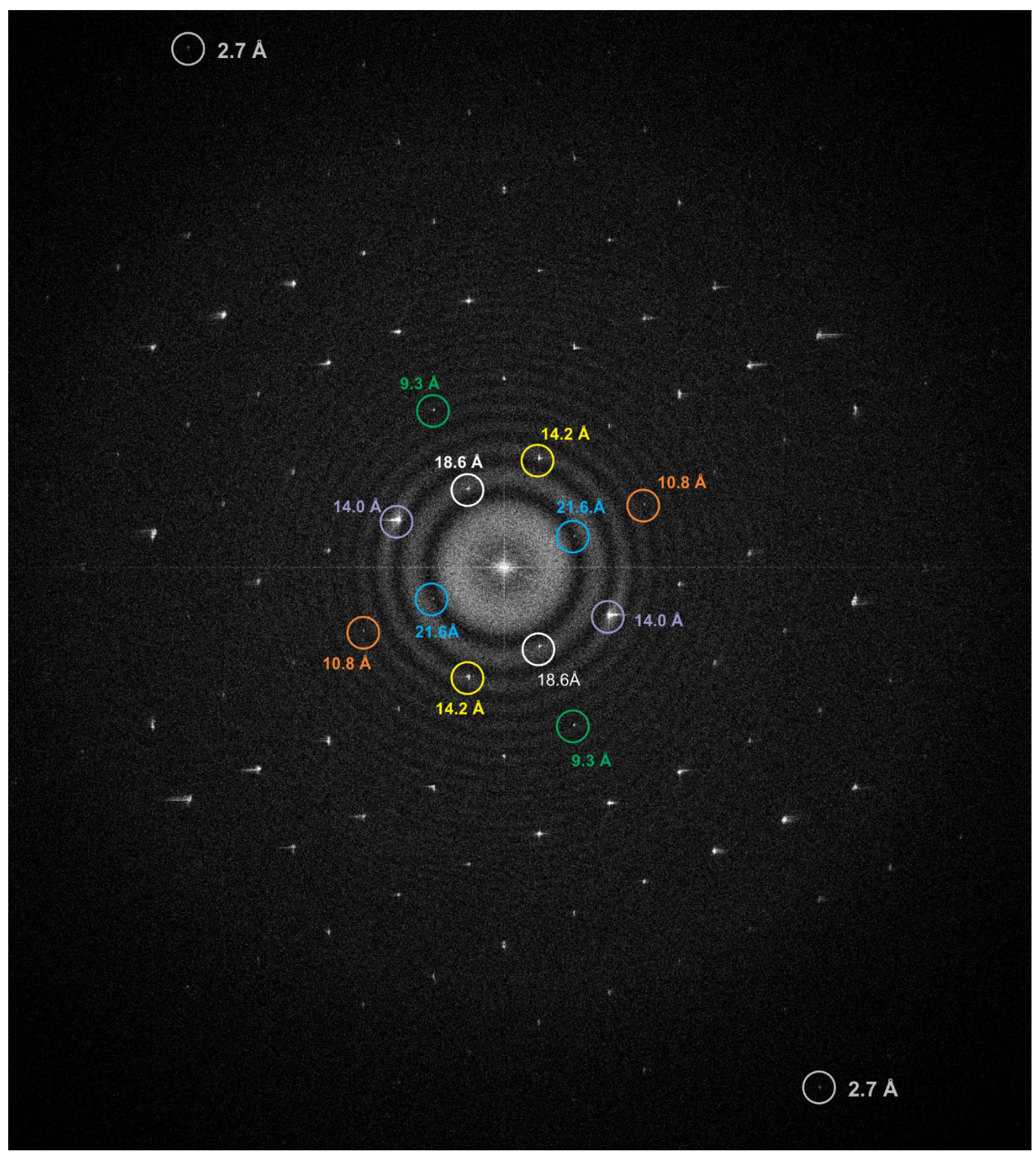

Figure S14. FFT of a cryo-EM image of the R6P6/P6E4 nanosheets. Structurally important Bragg spots are labeled with their corresponding $d$-spacing values. Note: averaged $d$-spacing values are reported. 

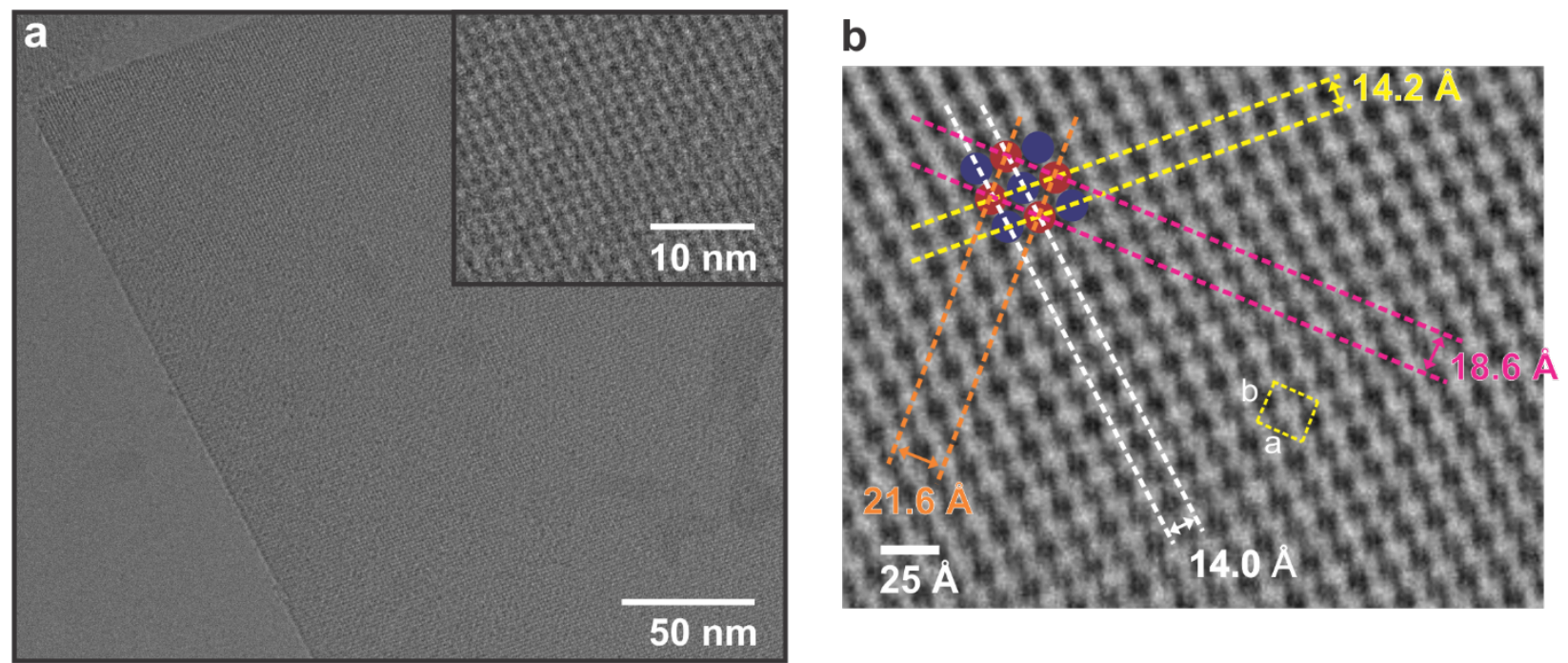

Figure S15. (a) Drift-corrected cryo-EM image of a R6P6/P6E4 nanosheet. Inset: high magnification image reveals the ordered 2D lattice. (b) Overlay of the lattice filtered cryo-EM image of a R6P6/P6E4 nanosheet over the original drift-corrected image revealing the 2D lattice. Labeled $d$-spacing values reflect the average measured distance from 10 nanocrystals. The 2D monoclinic unit cell is indicated with the associated lattice parameters "a" and "b".

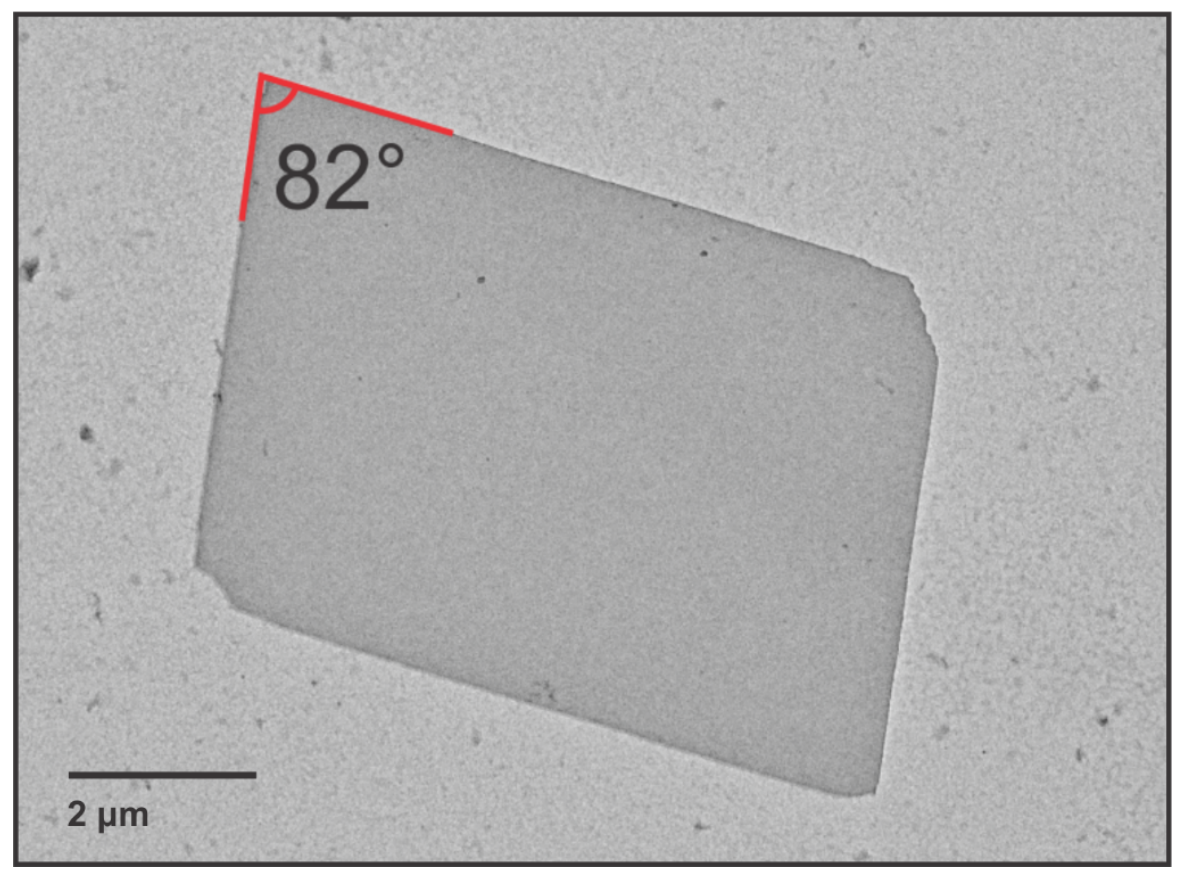

Figure S16. Parallelogram morphology of a R6P6/P6E4 nanosheet, in which two opposite corners exhibit an internal angle of ca. $82^{\circ}$. 


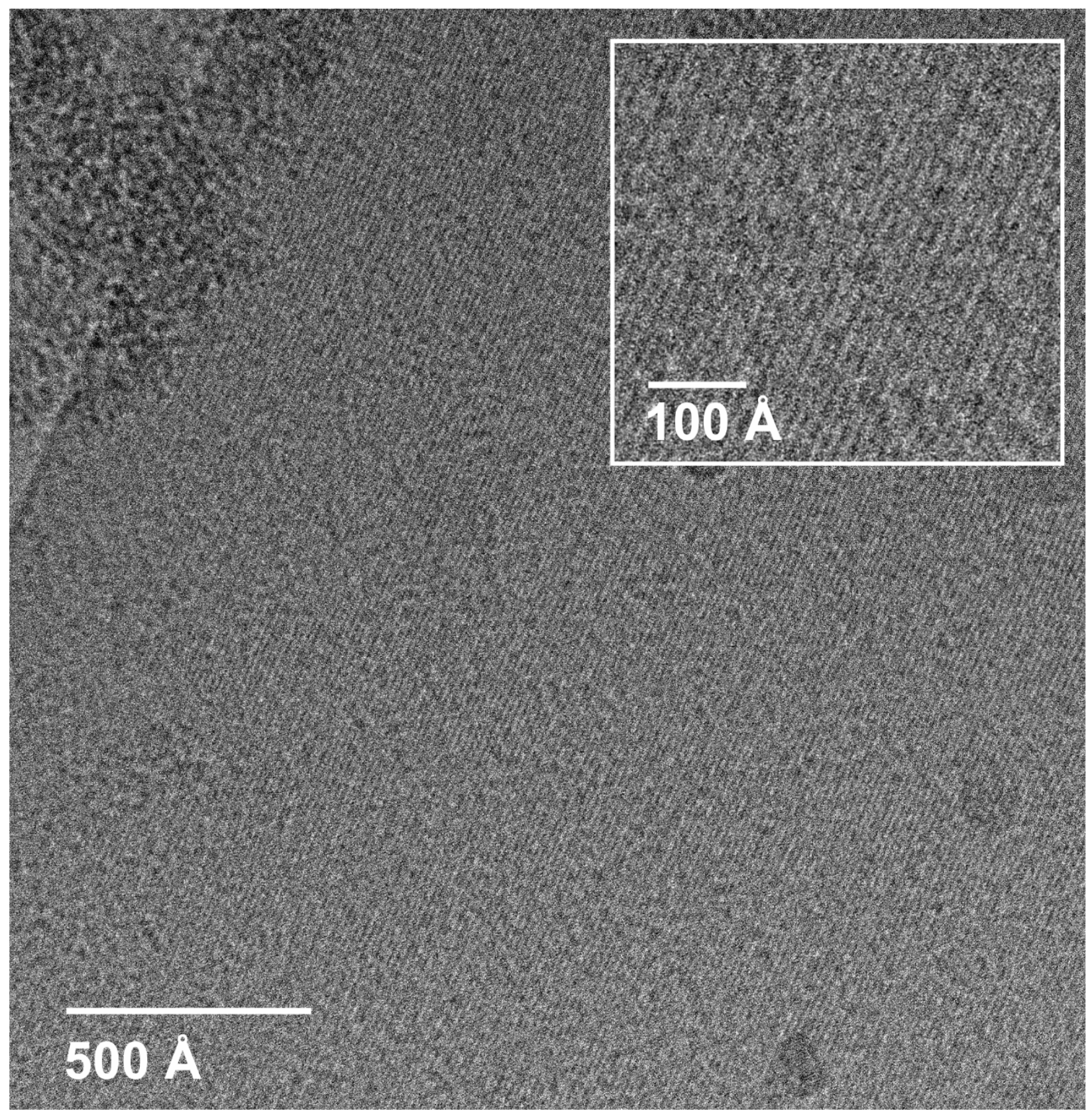

Figure S17. Unprocessed cryo-EM images revealing parallel "lines" as opposed to a grid-like tetragonal pattern typically observed for 2D layers of collagen triple helices. 

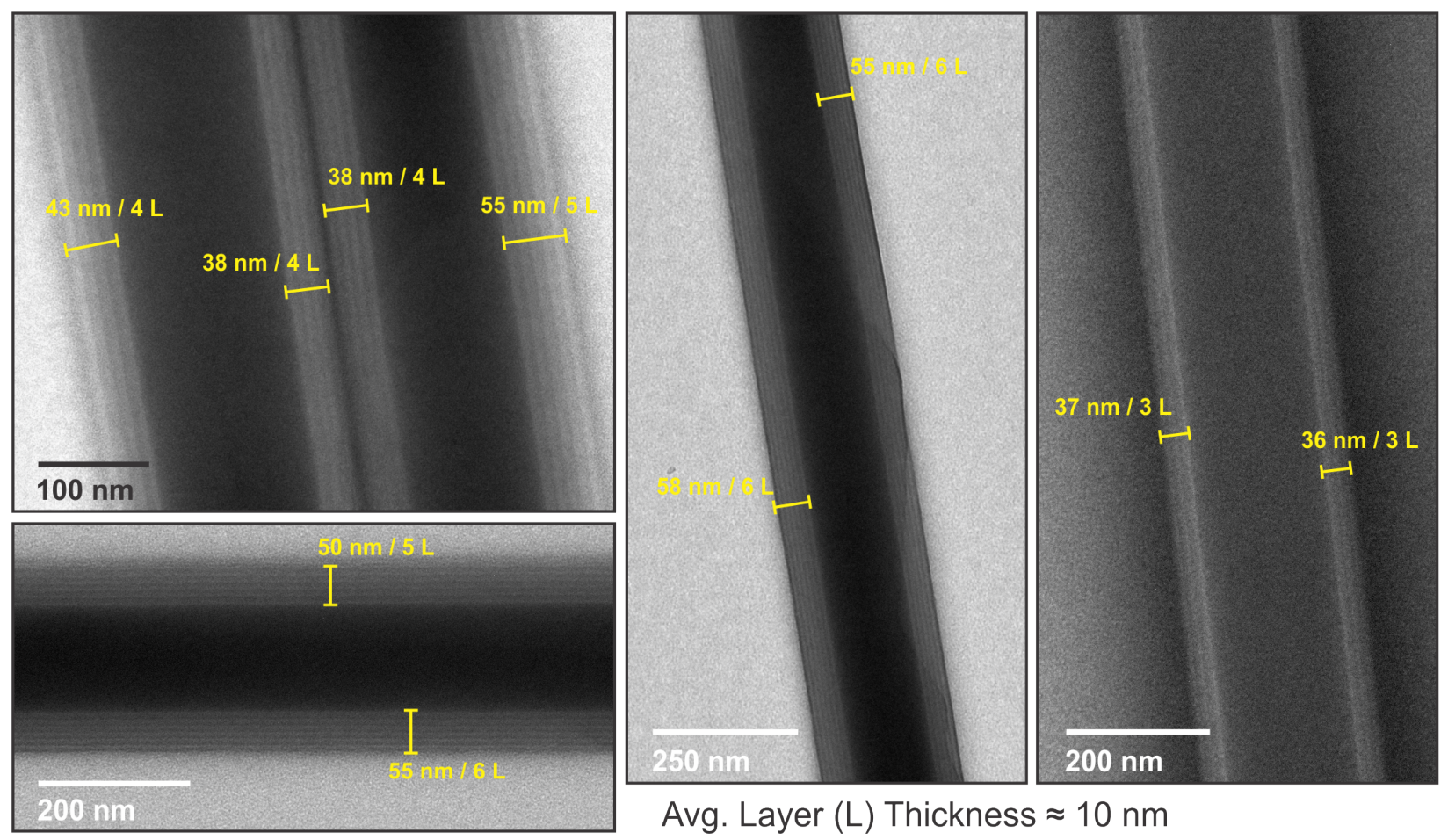

Avg. Layer (L) Thickness $\approx 10 \mathrm{~nm}$

Figure S18. Negatively stained TEM images of several representative R6P6/P6E4 tubes. Analysis of layer thicknesses observed within the tube walls reveals an average layer thickness of approximately $10 \mathrm{~nm}$. 

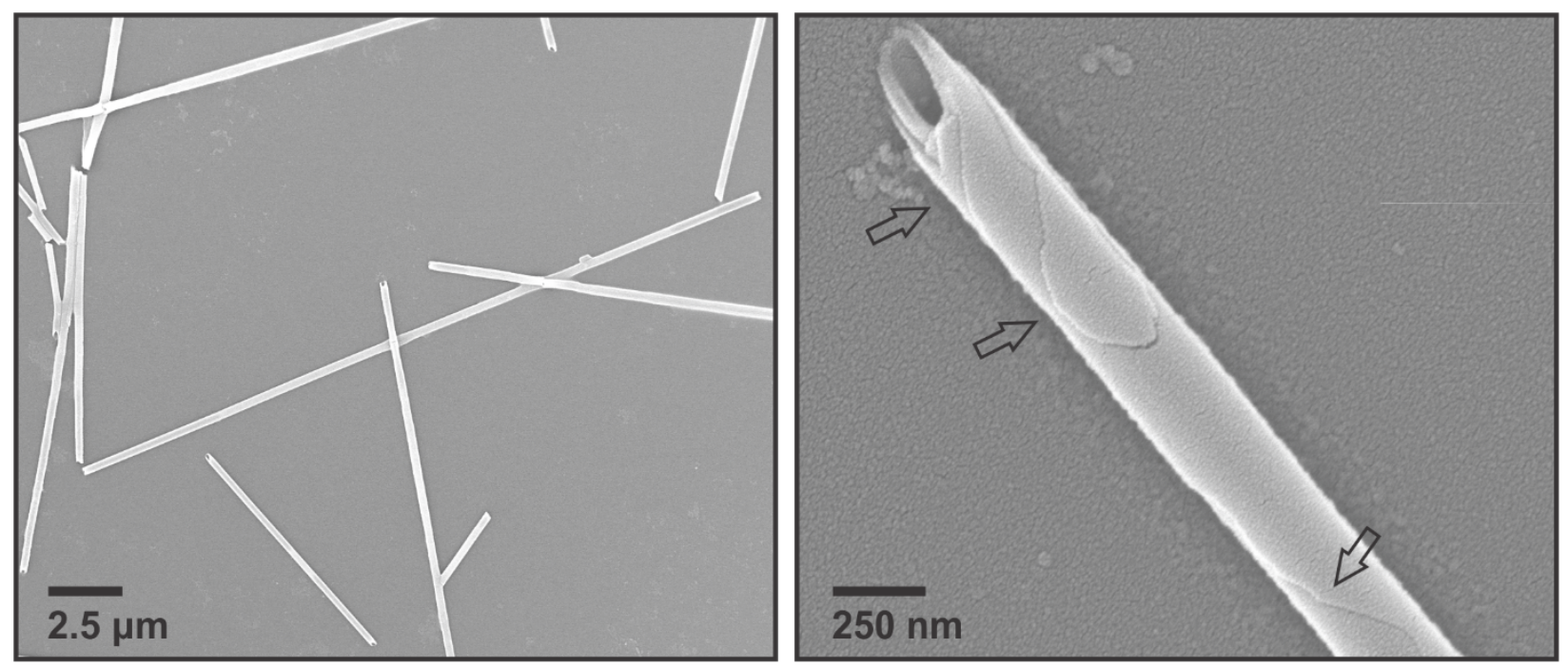

Figure S19. SEM micrographs of R6P6/P6E4 tubes assembled in TAPS buffer (20 mM, pH 8.0). Arrows indicate regions in which layers associated with individual scrolled layers can be detected. 


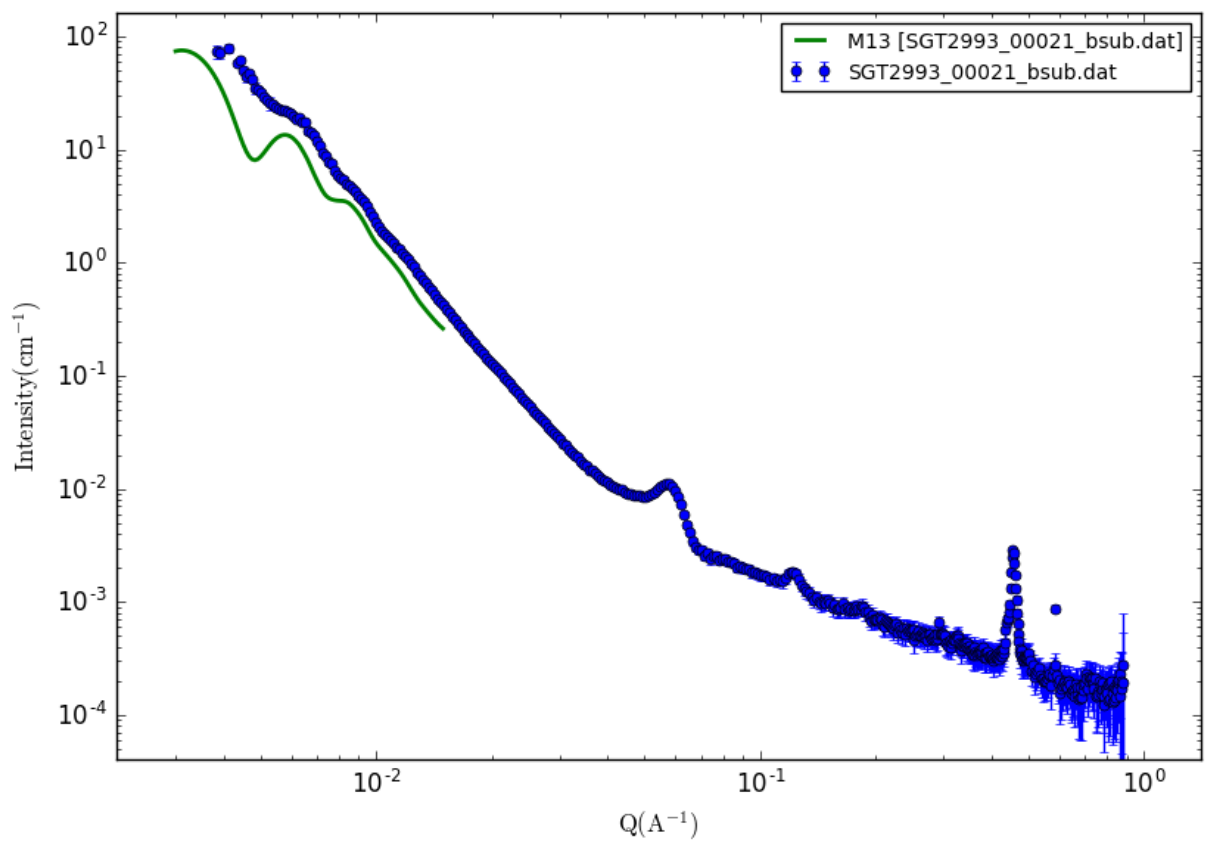

Figure S20. Fit of experimental synchrotron SAXS data (blue) for R6P6/P6E4 nanotubes (2 $\mathrm{mg} / \mathrm{mL})$ in TAPS buffer $(20 \mathrm{mM}, \mathrm{pH} 8.0)$ to a hollow cylinder model (green) with size polydispersity (inner radius $=95 \pm 12 \mathrm{~nm}$ and wall thickness $=37 \pm 4 \mathrm{~nm}$ ). Polydispersity of the sample attenuates the oscillation in the form factor region. Blue dots represent experiment data points of R6P6/P6E4 tubes. 


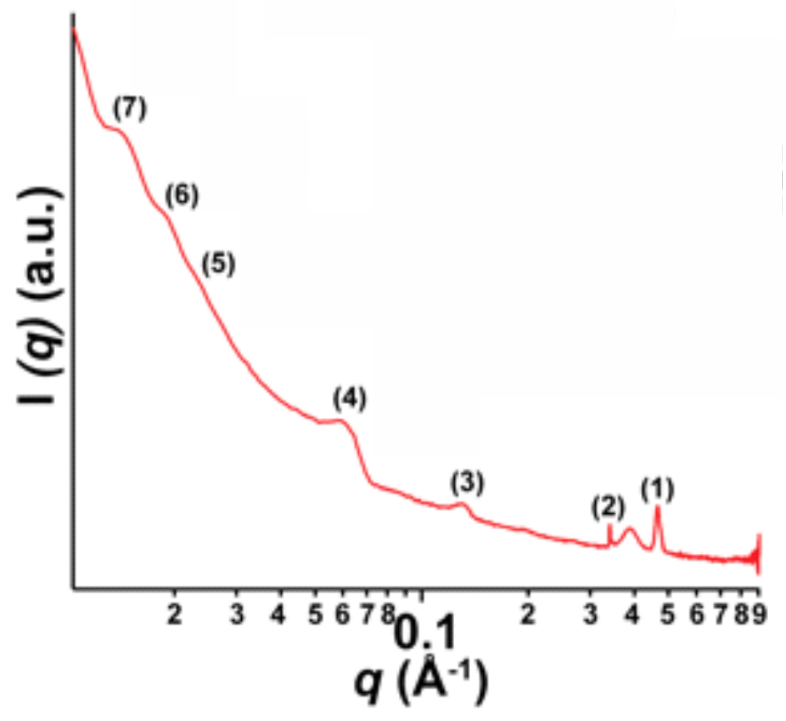

Figure S21. Synchrotron SAXS scattering profile of R4P6/P6E4 tubes $(1 \mathrm{mg} / \mathrm{mL})$ in MOP buffer (20 mM, pH 7.0). $d$-spacings: (1) $13.5 \AA$, (2) $18.4 \AA$, (3) 48.3 $\AA$, (4) 104.7. . Peaks (5), (6), and (7) are attributed to form factor scattering arising from tubular morphology with polydisperse cross-section. 


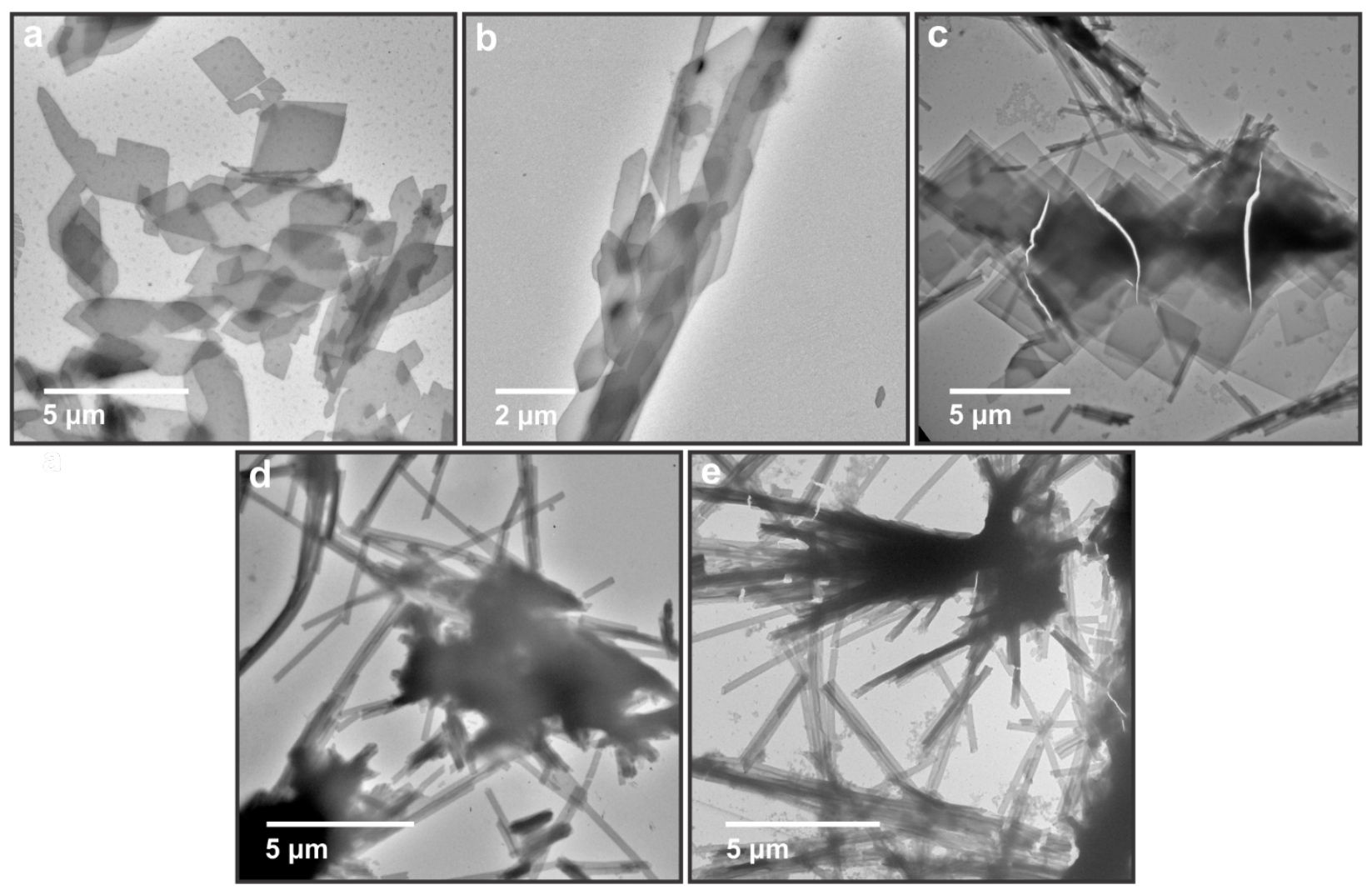

Figure S22. Stained TEM images of R6P6/R4P6/P6E4 assemblies in MOPS buffer (20 mM, pH 7.0) at different compositional ratios (R6P6/R4P6): (a) 90/10; (b) 75/25; (c) 50/50; (d) 25/75; and (e) $10 / 90$. 

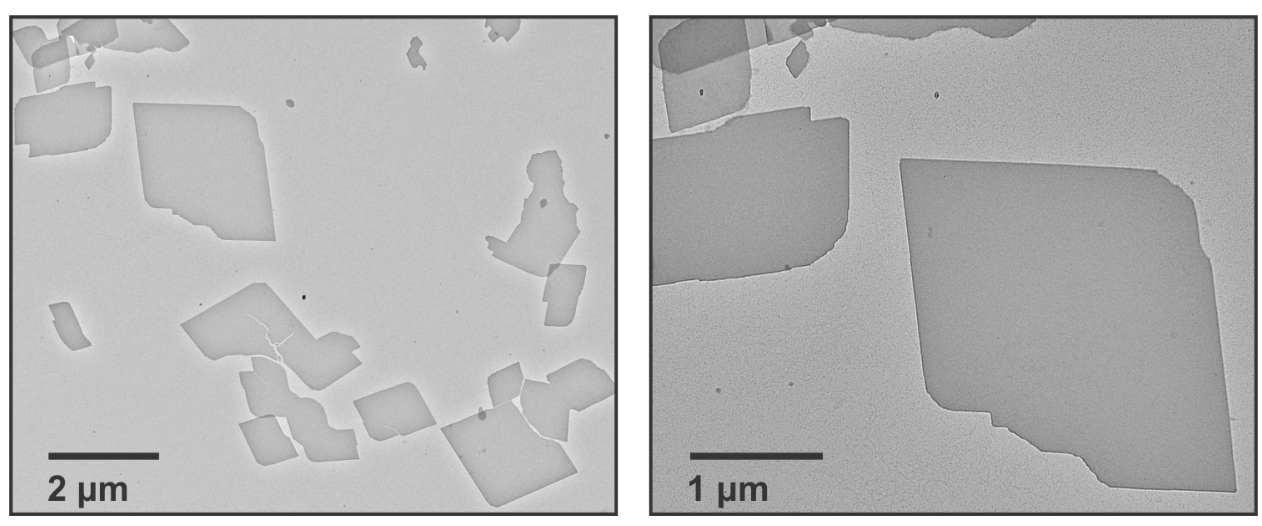

Figure S23. Stained TEM image of R6P6/P6E4 grown in MOPS buffer (20 mM, pH 7.0). These nanosheets were used as the starting materials for the in situ morphology transition study.
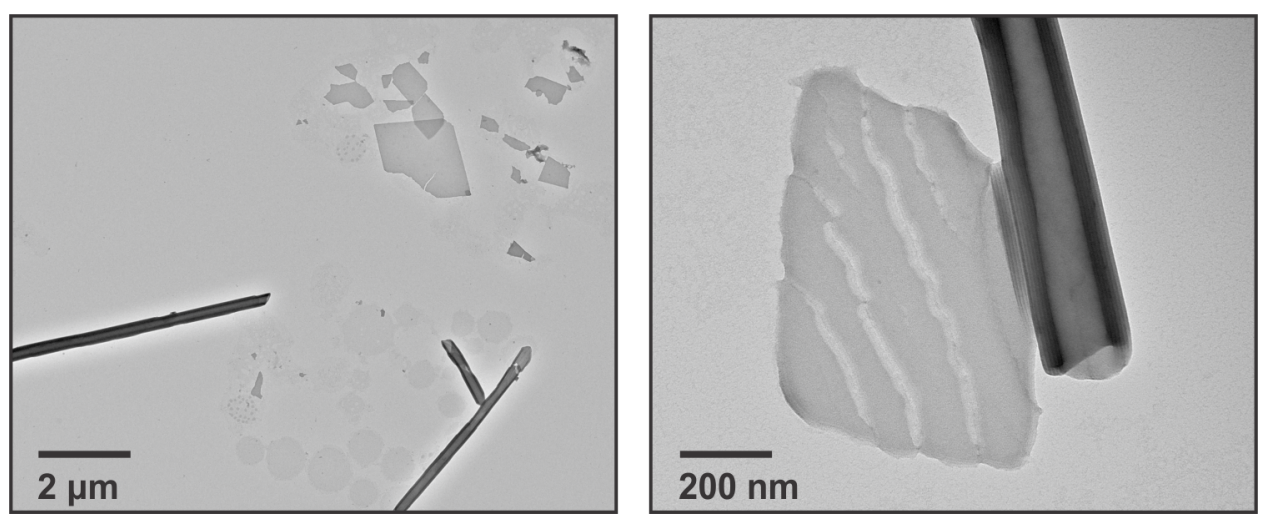

Figure S24. Stained TEM images of R6P6/P6E4 assemblies taken after 1 week in TAPS buffer (20 mM, pH 8.5). 

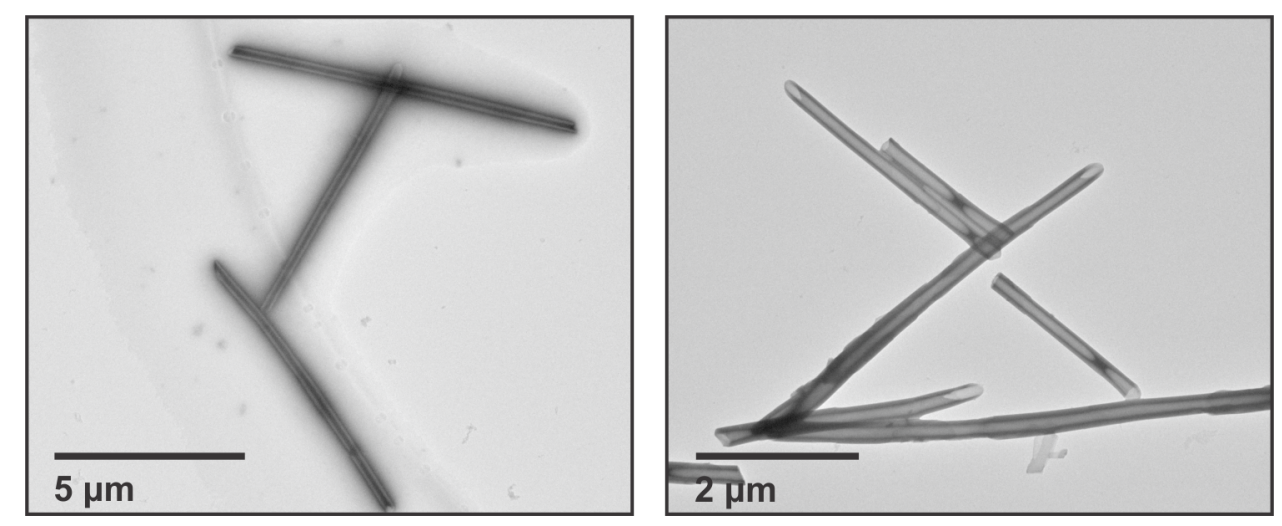

Figure S25. Stained TEM images of R6P6/P6E4 tubes taken after 2 weeks in TAPS buffer (20 $\mathrm{mM}, \mathrm{pH} 8.5)$.
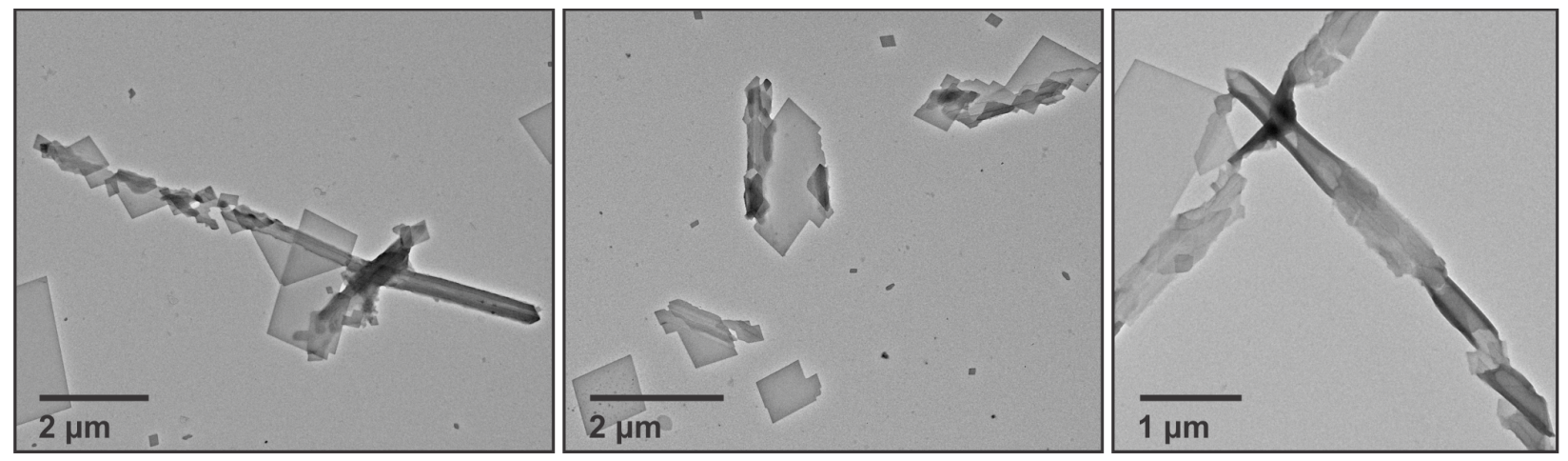

Figure S26. Stained TEM images of R6P6/P6E4 assemblies after 3 days in MOPS buffer (20 mM, $\mathrm{pH} 7.0)$. 


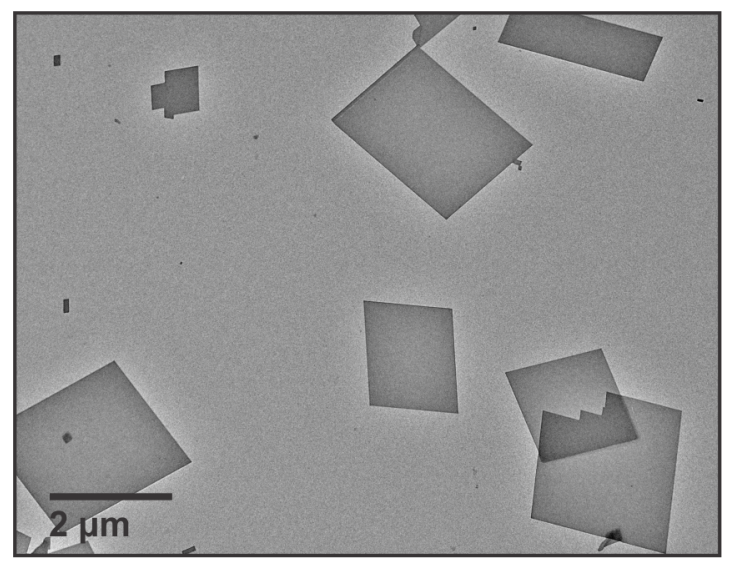

Figure S27. Stained TEM image of R6P6/P6E4 nanosheets obtained after 1 week in MOPS buffer (20 mM, pH 7.0). Nanosheets were observed at the conclusion of the in situ morphology transition study, confirming that the morphological transition from sheets to tubes is reversible. 

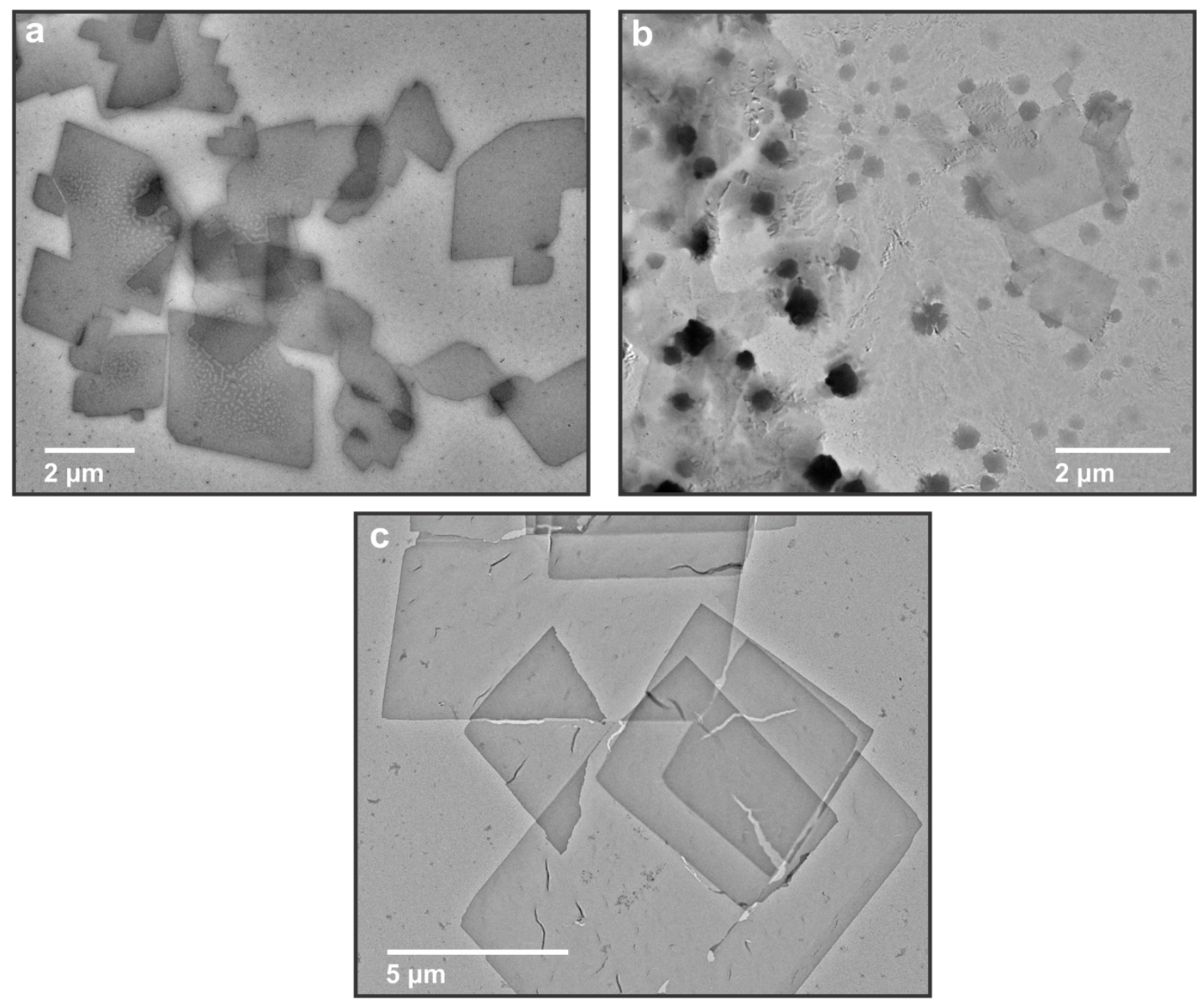

Figure S28. Stained TEM images of R6P6/P6E4 nanosheets incubated in the presence of (a) 10 $\mathrm{mM} \mathrm{NaCl}$ and (b) $100 \mathrm{mM} \mathrm{NaCl}$ after 2 days and 1 week, respectively. $\mathrm{NaCl}$ was introduced to pre-formed R6P6/P6E4 nanosheets in $20 \mathrm{mM}$ MOPS buffer (pH 7.0). Salt contamination on the surface of the grid is noticeable at $100 \mathrm{mM} \mathrm{NaCl}$ concentration. (c) Stained TEM image of R6P6/P6E4 nanosheets that were formed in the presence of $100 \mathrm{mM} \mathrm{NaCl}$ in $20 \mathrm{mM}$ MOPS buffer $(\mathrm{pH} 7.0)$. 
a

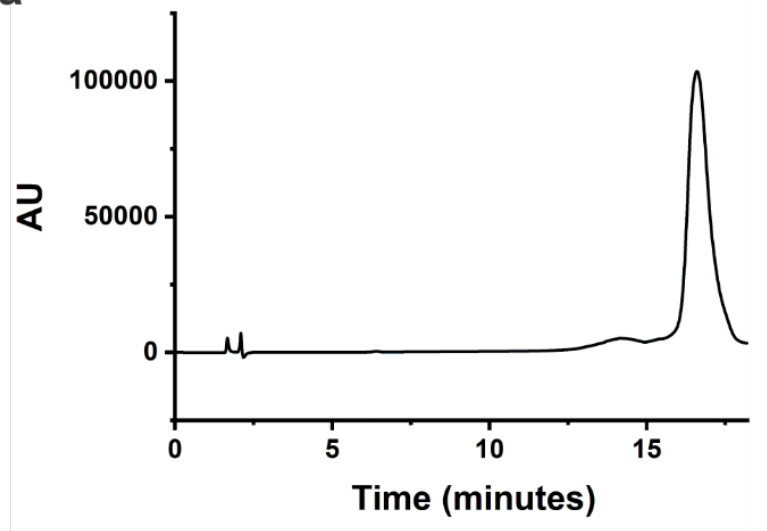

b

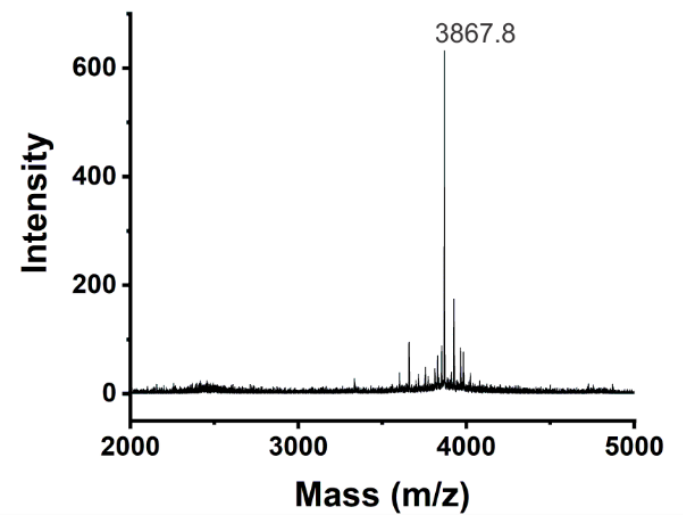

Figure S29. (a) HPLC trace of b-R6P6. (b) MALDI-TOF mass spectrum of purified b-R6P6, m/z $=3867.8\left(\mathrm{M}+\mathrm{H}^{+}\right)$.

\begin{tabular}{c|c|c|} 
& pl & pl \\
(https://web.expasy.org/compute_pi/) & (http://isoelectric.org/) \\
\hline R4P6 & 12.48 & 12.32 \\
\hline R6P6 & 12.70 & 12.54 \\
\hline R3P6 & 12.30 & 12.14 \\
\hline P6E4 & 3.58 & 3.28 \\
\hline
\end{tabular}

Table S1. Estimated isoelectric point, pI, values of CMPs employed in this work. Estimated pI values were obtained from ExPASy Compute $\mathrm{pI} / \mathrm{MW}$ tool ${ }^{1}$ and IPC - Isoelectric Point Calculator. ${ }^{2}$ 


\begin{tabular}{c|c|c|} 
& $\begin{array}{c}\mathrm{pl} \\
\text { (https://web.expasy.org/compute_pi/) }\end{array}$ & $\begin{array}{c}\mathrm{pl} \\
\text { (http://isoelectric.org/) }\end{array}$ \\
\hline R4P6/P6E4 & 6.72 & 6.77 \\
\hline R6P6/P6E4 & 11.30 & 11.15 \\
\hline R3P6/P6E4 & 4.95 & 4.90 \\
\hline
\end{tabular}

Table S2. Estimated isoelectric point, pI, values for co-assemblies of CMP assemblies employed in this work. The $\mathrm{pI}$ values were estimated using a procedure in which the two peptides were combined into a single sequence. Estimated pI values were obtained from ExPASy Compute $\mathrm{pI} / \mathrm{MW}$ tool $^{1}$ and IPC - Isoelectric Point Calculator. ${ }^{2}$

\section{References:}

1. Gasteiger, E.; Hoogland, C.; Gattiker, A.; Duvaud, S.; Wilkins, M. R.; Appel, R. D.; Bairoch, A. Protein Identification and Analysis Tools on the ExPASy Server; Walker, J. M.; Humana Press. 2005; DOI: $10.1385 / 1592598900$

2. Kozlowski, L. P. IPC - Isoelectric Point Calculator. Biol. Direct 11, 55 (2016). https://doi.org/10.1186/s13062-016-0159-9 